\title{
Terminal phosphinidene complex adducts with neutral and anionic $O$ - donors and halides, and the search for a differentiating bonding descriptor
}

\section{Arturo Espinosa Ferao, ${ }^{* a}$ Antonio García Alcaraz, ${ }^{\text {a Salvador Zaragoza Noguera, }}{ }^{\text {a }}$ Rainer Streubel*b}

\footnotetext{
a Departamento Química Orgánica, Facultad de Química, Campus de Espinardo, Universidad de Murcia, 30100 Murcia (Spain), E-mail: artuesp@um.es.

b Institut für Anorganische Chemie der Rheinischen Friedrich-Wilhelms-Universität Bonn, GerhardDomagk-Straße 1, 53121 Bonn (Germany), E-mail: r.streubel@uni-bonn.de.
}

\section{Table of contents}

Figure S1: Computed structure for adduct 1q,s showing bond and ring critical points, as well as bond paths.

page

Figure S2: Plot of LBO versus WBI for adducts 1.

Figure S3: Plot of $\nabla^{2} \rho$ versus the dative covalent energy $D_{1} E_{1}$ for adducts 1.

Figure S4: Variation of $\nabla^{2} \rho$ along the O-P bond paths for $\mathrm{CO}_{2}$ and $\mathrm{N}_{2} \mathrm{O}$ adducts 1g,o. S3

Figure S5: Variation of $\nabla^{2} \rho$ along the O-P bond paths for cyclic ether adducts $\mathbf{1 h}-\mathbf{j}$. S4

Figure S6: Variation of $\nabla^{2} \rho$ along the $\mathrm{Me}_{3} \mathrm{~N}-\mathrm{O}$ and $\mathrm{O}-\mathrm{P}$ bond paths for $\mathrm{Me}_{3} \mathrm{NO}$ adduct $\mathbf{1 k}$. S5

Figure S7: Variation of $\nabla^{2} \rho$ along the $\mathrm{Me}_{3} \mathrm{P}-\mathrm{O}$ and $\mathrm{O}-\mathrm{P}$ bond paths for $\mathrm{Me}_{3} \mathrm{PO}$ adduct $\mathbf{1 l}$. S5

Figure S8: Variation of $\nabla^{2} \rho$ along the $\mathrm{Me}_{2} \mathrm{~S}-\mathrm{O}$ and O-P bond paths for $\mathrm{Me}_{2} \mathrm{SO}$ adduct $\mathbf{1 m}$. S6

Figure S9: Variation of $\nabla^{2} \rho$ along the O-P bond paths for $\mathrm{MeO}^{-}$and ${ }^{\mathrm{s}} \mathrm{O}_{2}$ adducts 1e,n. S6

Figure S10: Variation of $\nabla^{2} \rho$ along the halogen-P bond paths for halide adducts 1a-d. $\quad$ S7

Figure S11: Variation of $\nabla^{2} \rho$ along the O-P or N-P bond paths for nitric oxide adducts 1p-s. S8

Calculated structures: Cartesian coordinates and energies for all minima and transition states. S9 


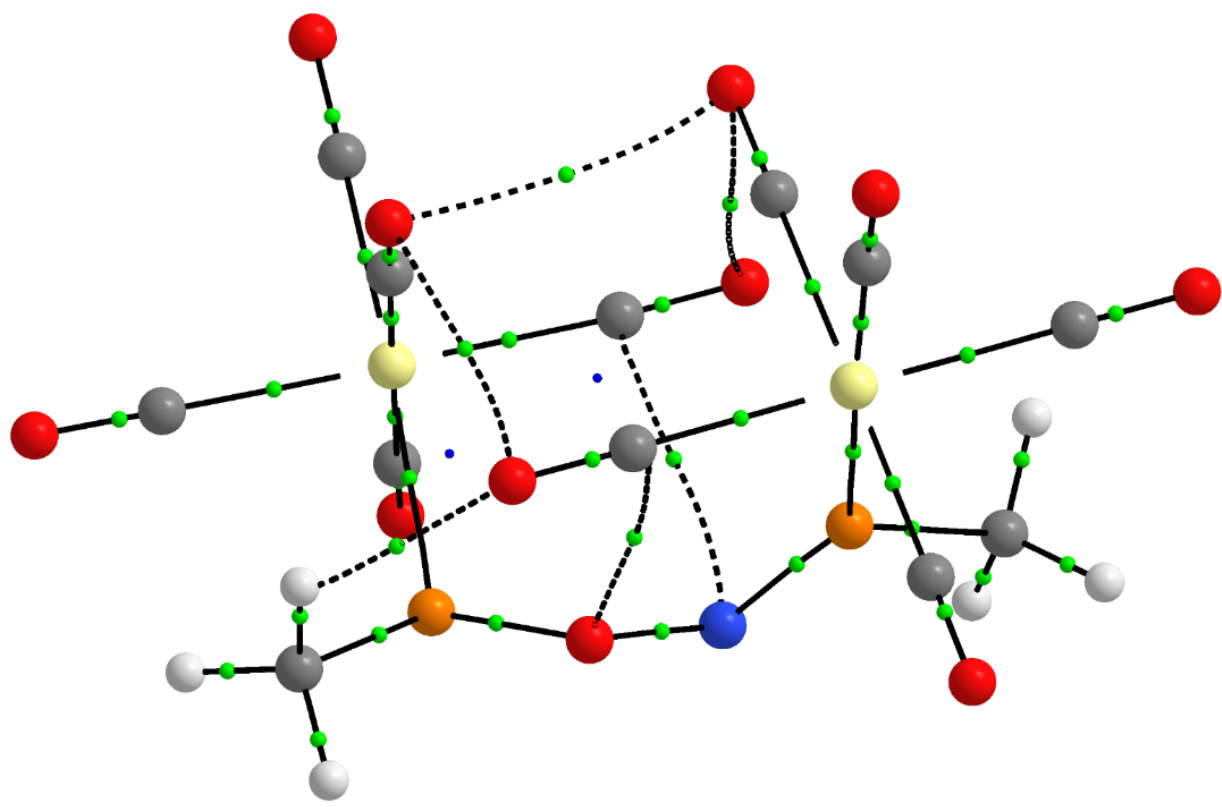

Figure S1. Computed (B3LYP-D3/def2-TZVP(еср)) structure for adduct 1q,s showing bond (small green spheres) and ring critical points (small dark blue spheres), as well as bond paths. 


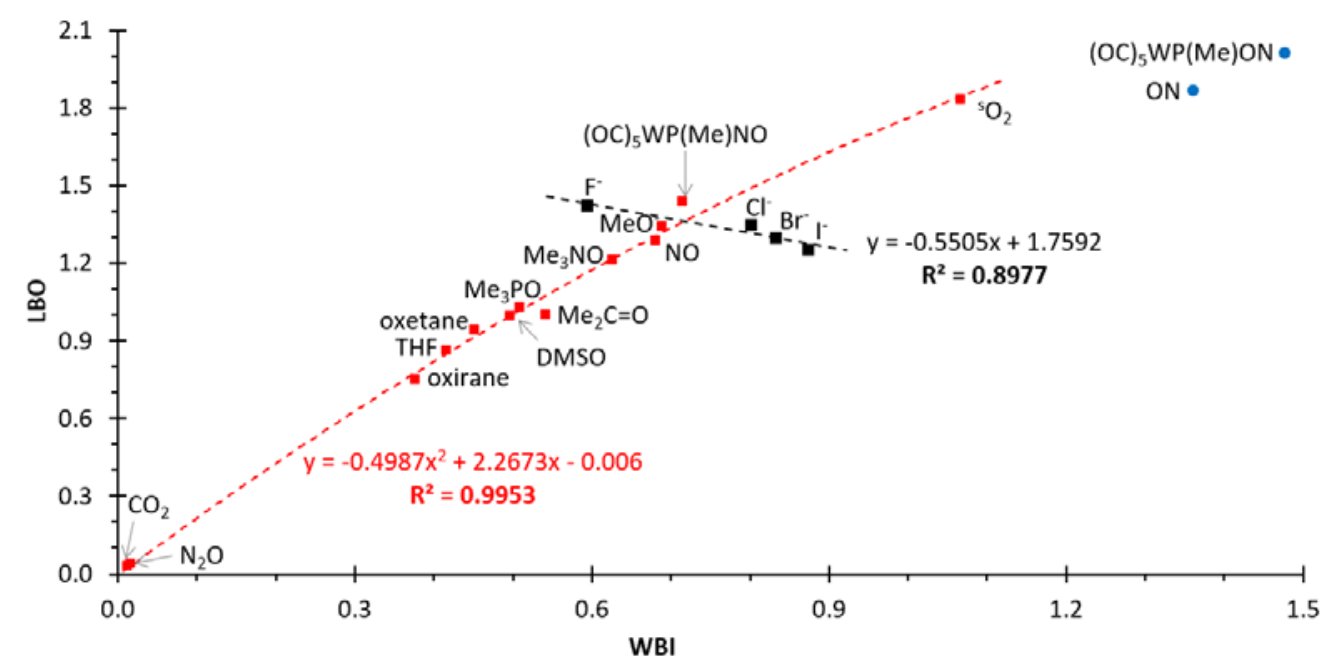

Figure S2. Plot of LBO versus WBI for adducts 1.

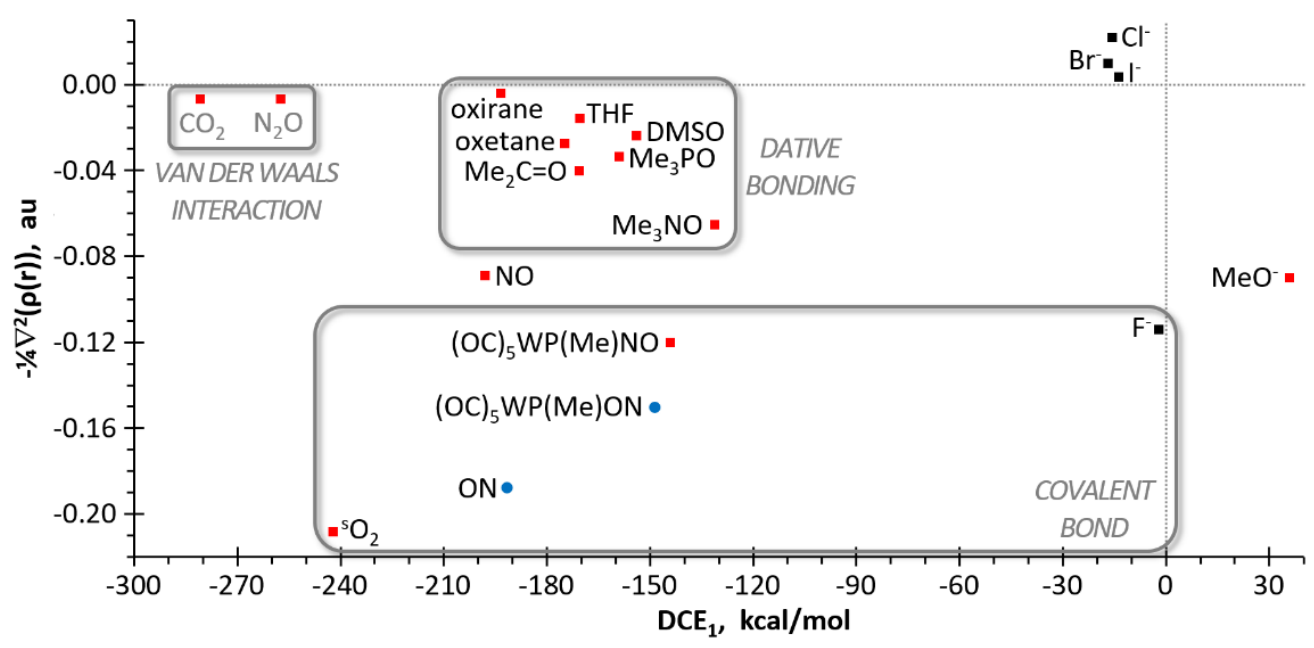

Figure S3. Plot of Laplacian of $\rho(r)$ versus the dative covalent energy DCE 1 for adducts 1.
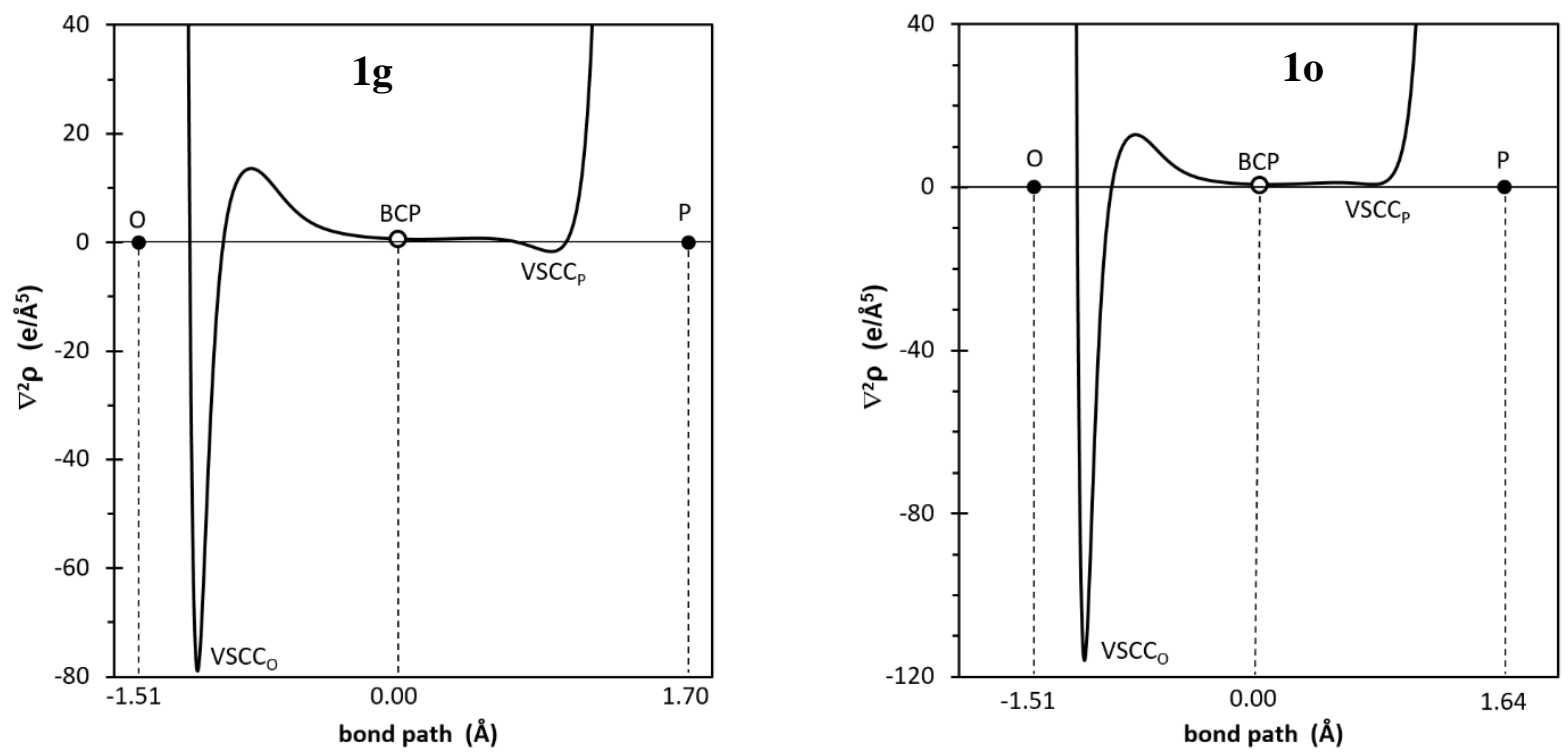

Figure S4. Computed (B3LYP-D3/def2-TZVPPecp//B3LYP-D3/def2-TZVPecp) variation of the Laplacian of electron density $\nabla^{2} \rho$ for $\mathbf{1 g}\left(\mathrm{L}: \mathrm{CO}_{2}\right.$, left) and $\mathbf{1 0}\left(\mathrm{L}: \mathrm{N}_{2} \mathrm{O}\right.$, right) along the O-P bond paths. 

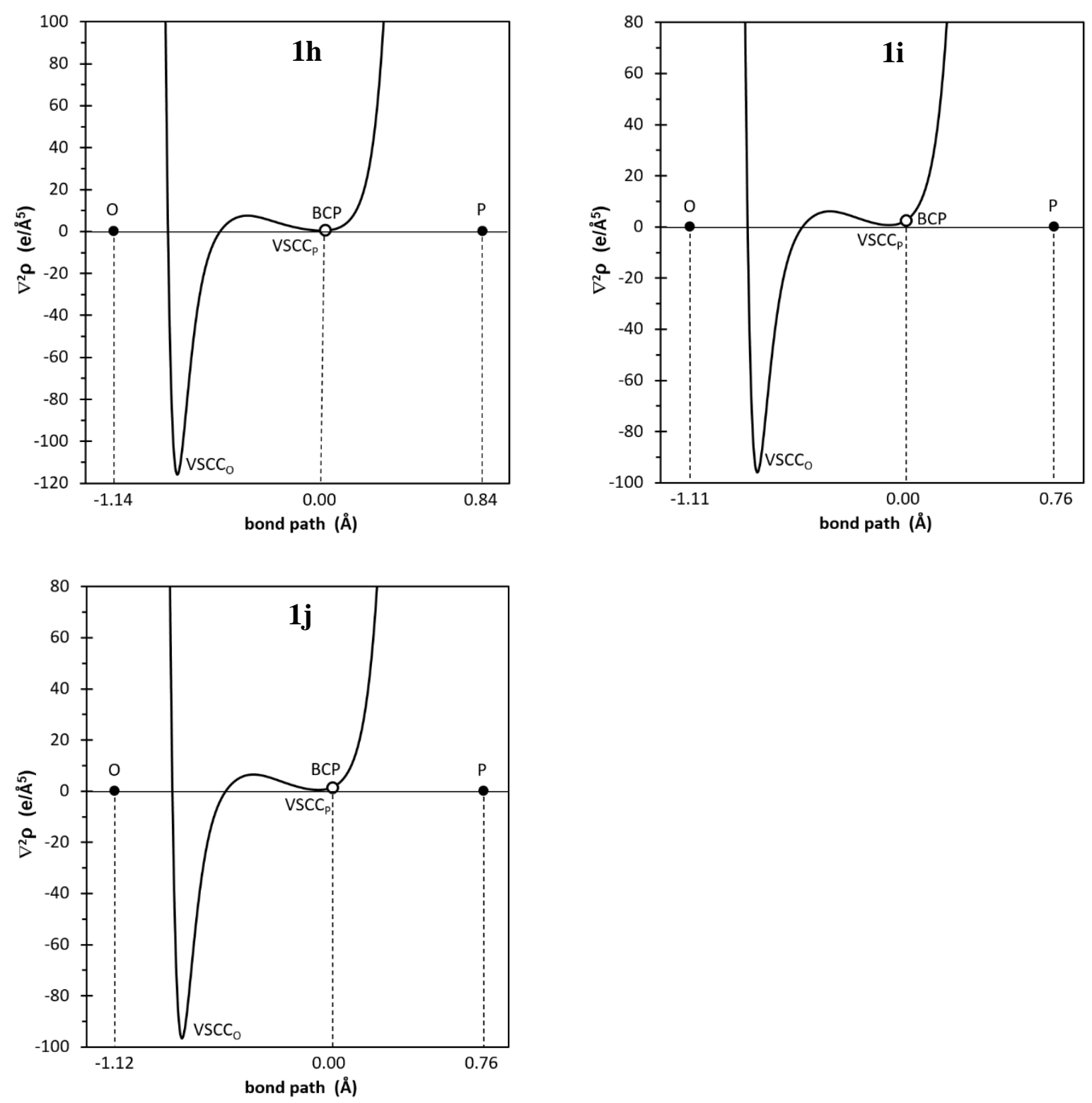

Figure S5. Computed (B3LYP-D3/def2-TZVPPecp//B3LYP-D3/def2-TZVPecp) variation of the Laplacian of electron density $\nabla^{2} \rho$ for adducts derived from cyclic ethers $\mathbf{1 h}-\mathbf{j}$ (L: oxirane, oxetane and THF, respectively) along the O-P bond paths. 


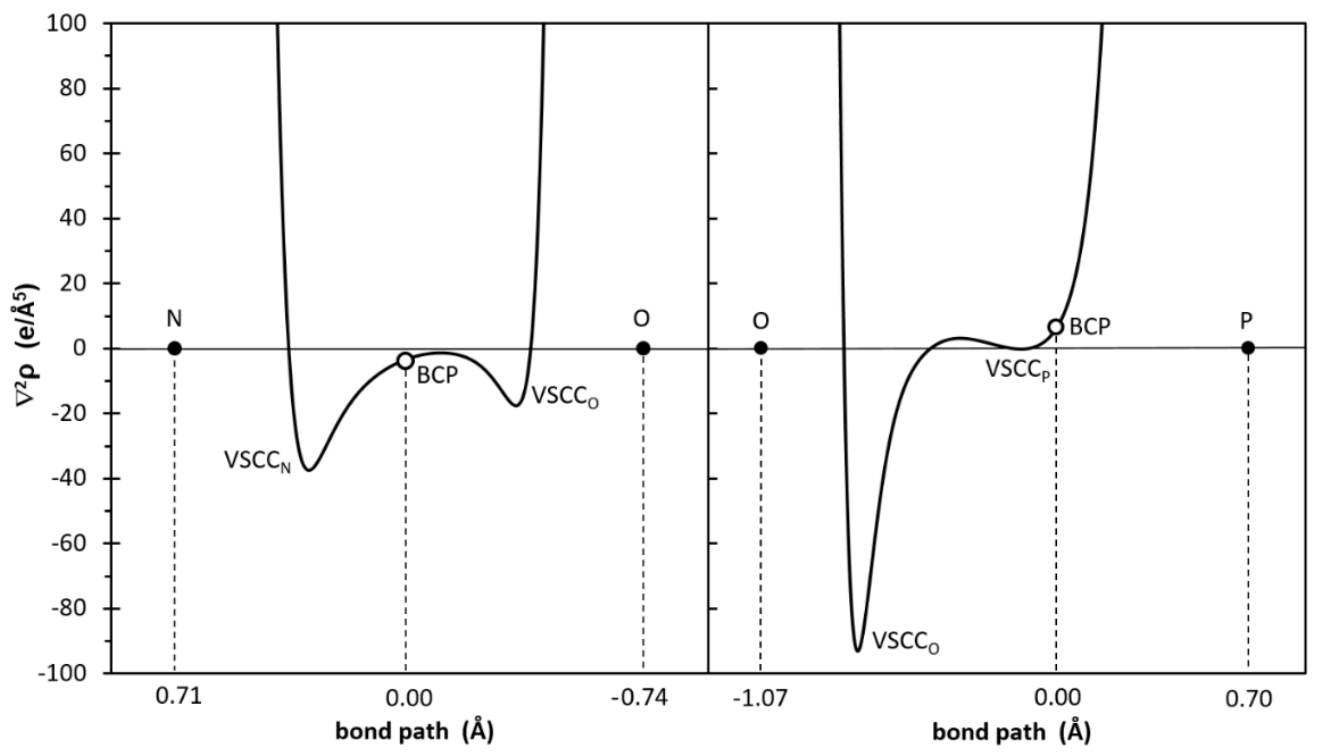

Figure S6. Computed (B3LYP-D3/def2-TZVPPecp//B3LYP-D3/def2-TZVPecp) variation of the Laplacian of electron density $\nabla^{2} \rho$ for adducts derived from trimethylamine oxide (1k) along the $\mathrm{Me}_{3} \mathrm{~N}$ $\mathrm{O}$ (left) and O-P (right) bond paths.

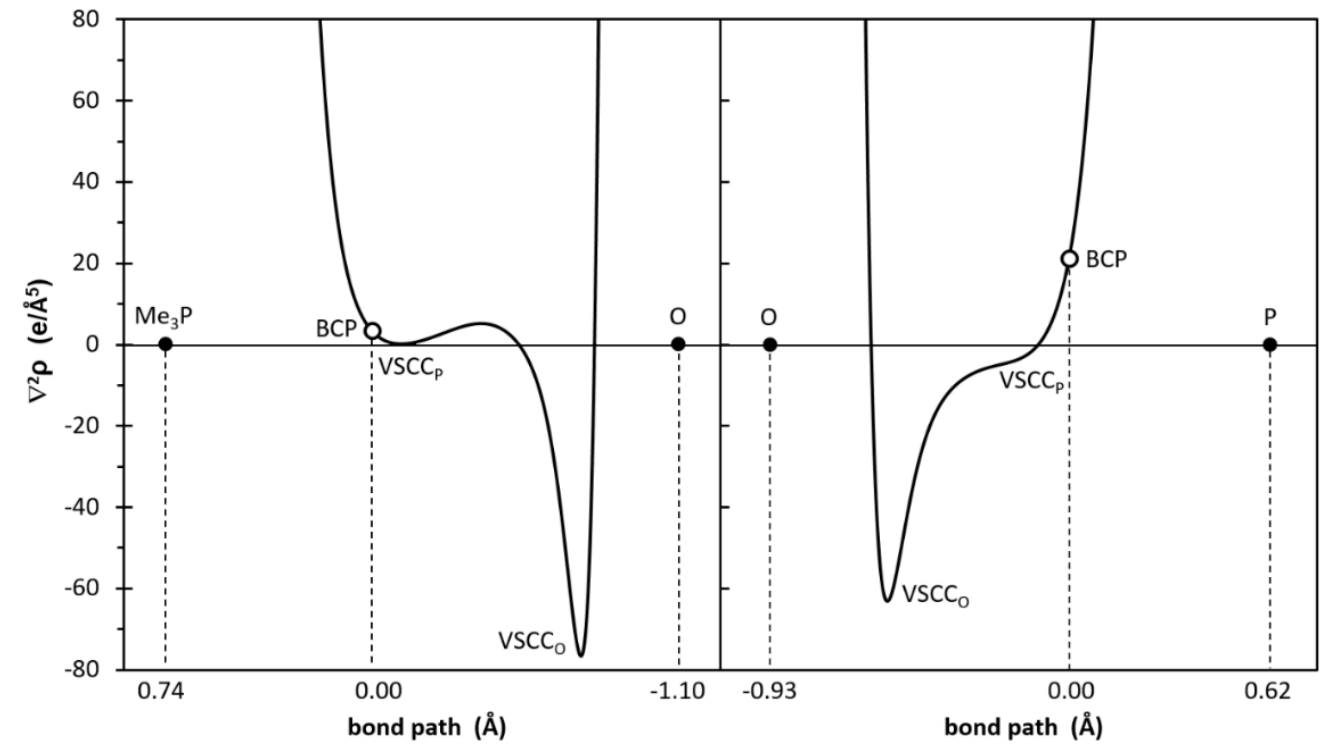

Figure S7. Computed (B3LYP-D3/def2-TZVPPecp//B3LYP-D3/def2-TZVPecp) variation of the Laplacian of electron density $\nabla^{2} \rho$ for adducts derived from trimethylphosphane oxide (11) along the $\mathrm{Me}_{3} \mathrm{P}-\mathrm{O}$ (left) and O-P (right) bond paths. 


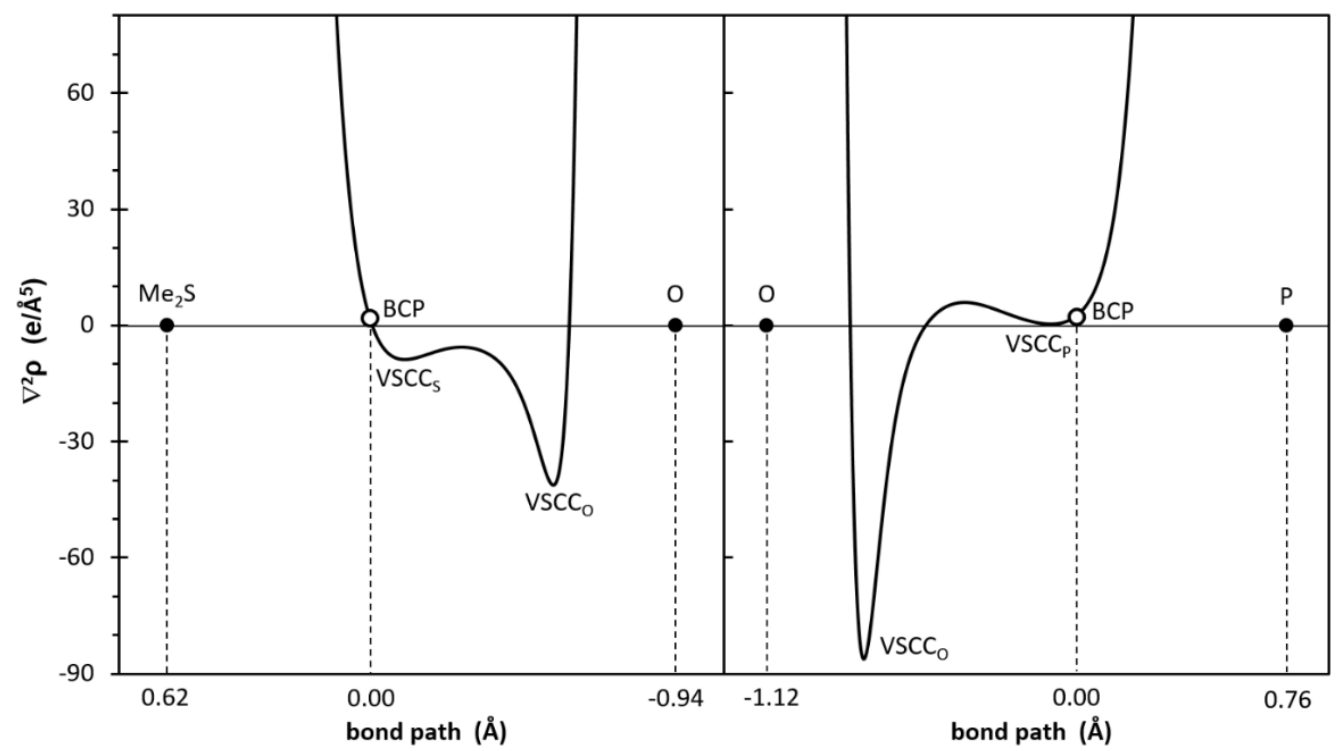

Figure S8. Computed (B3LYP-D3/def2-TZVPPecp//B3LYP-D3/def2-TZVPecp) variation of the Laplacian of electron density $\nabla^{2} \rho$ for adducts derived from dimethylsulfoxide (1m) along the $\mathrm{Me}_{2} \mathrm{~S}-\mathrm{O}$ (left) and O-P (right) bond paths.
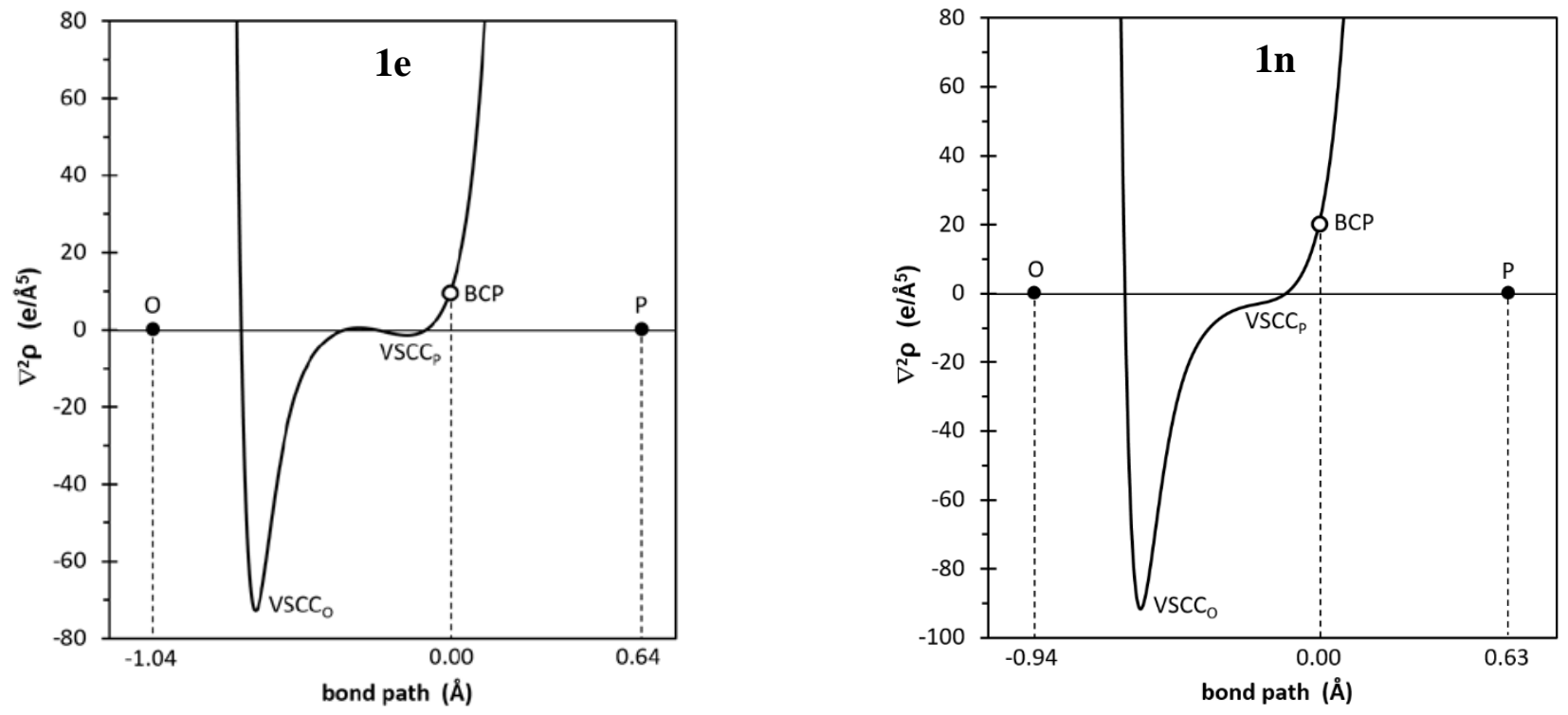

Figure S9. Computed (B3LYP-D3/def2-TZVPPecp//B3LYP-D3/def2-TZVPecp) variation of the Laplacian of electron density $\nabla^{2} \rho$ for $1 \mathbf{e}\left(\mathrm{L}: \mathrm{MeO}^{-}\right)$and $\mathbf{1 n}\left(\mathrm{L}^{\left.{ }^{\mathrm{S}} \mathrm{O}_{2}\right)}\right.$ along the O-P bond paths. 

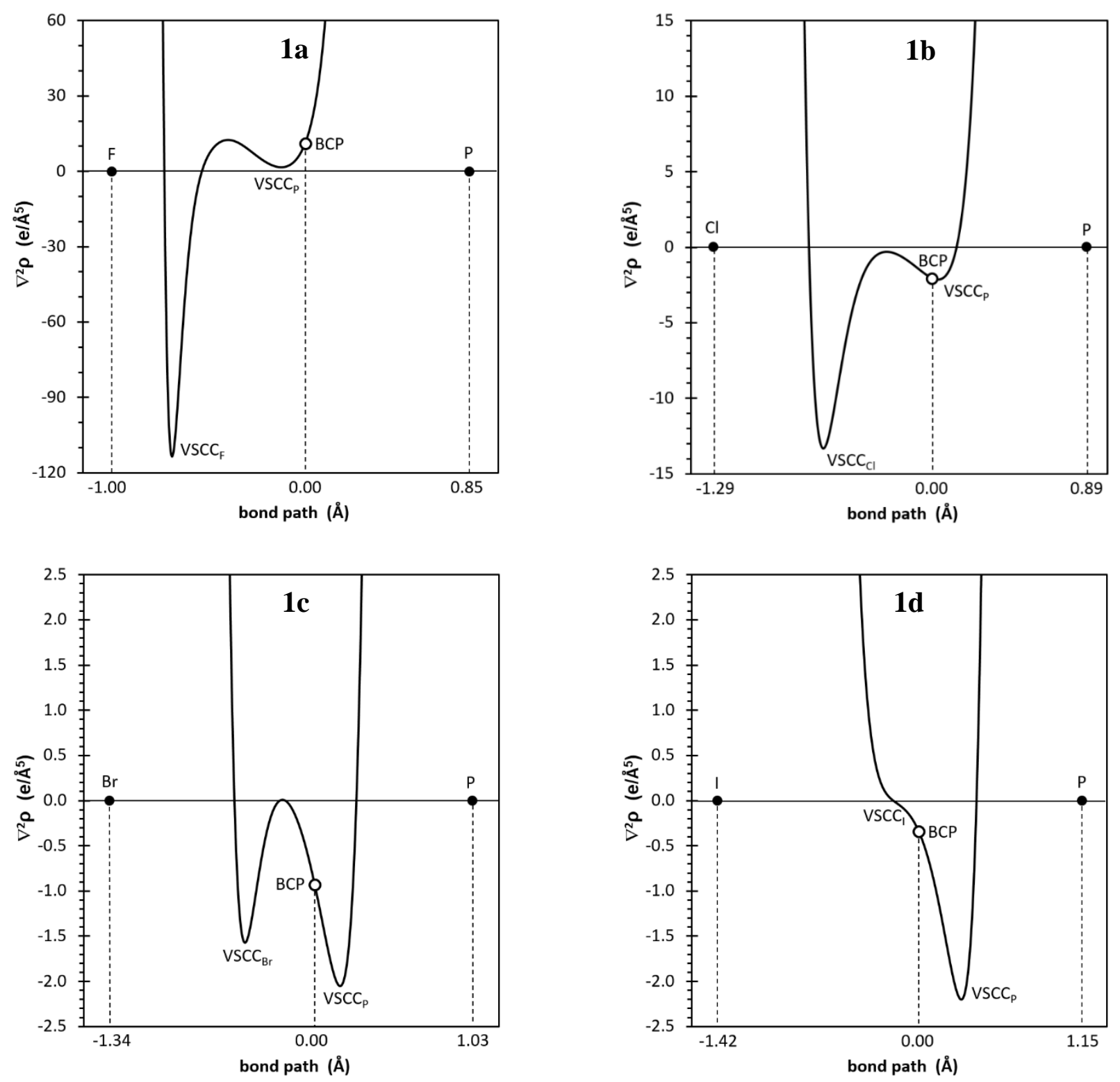

Figure S10. Computed (B3LYP-D3/def2-TZVPPecp//B3LYP-D3/def2-TZVPecp) variation of the Laplacian of electron density $\nabla^{2} \rho$ for adducts derived from halides 1a-d ( $\mathrm{L}^{-} \mathrm{F}^{-}, \mathrm{Cl}^{-}, \mathrm{Br}^{-}$and $\mathrm{I}^{-}$, respectively) along the halogen-P bond paths. 

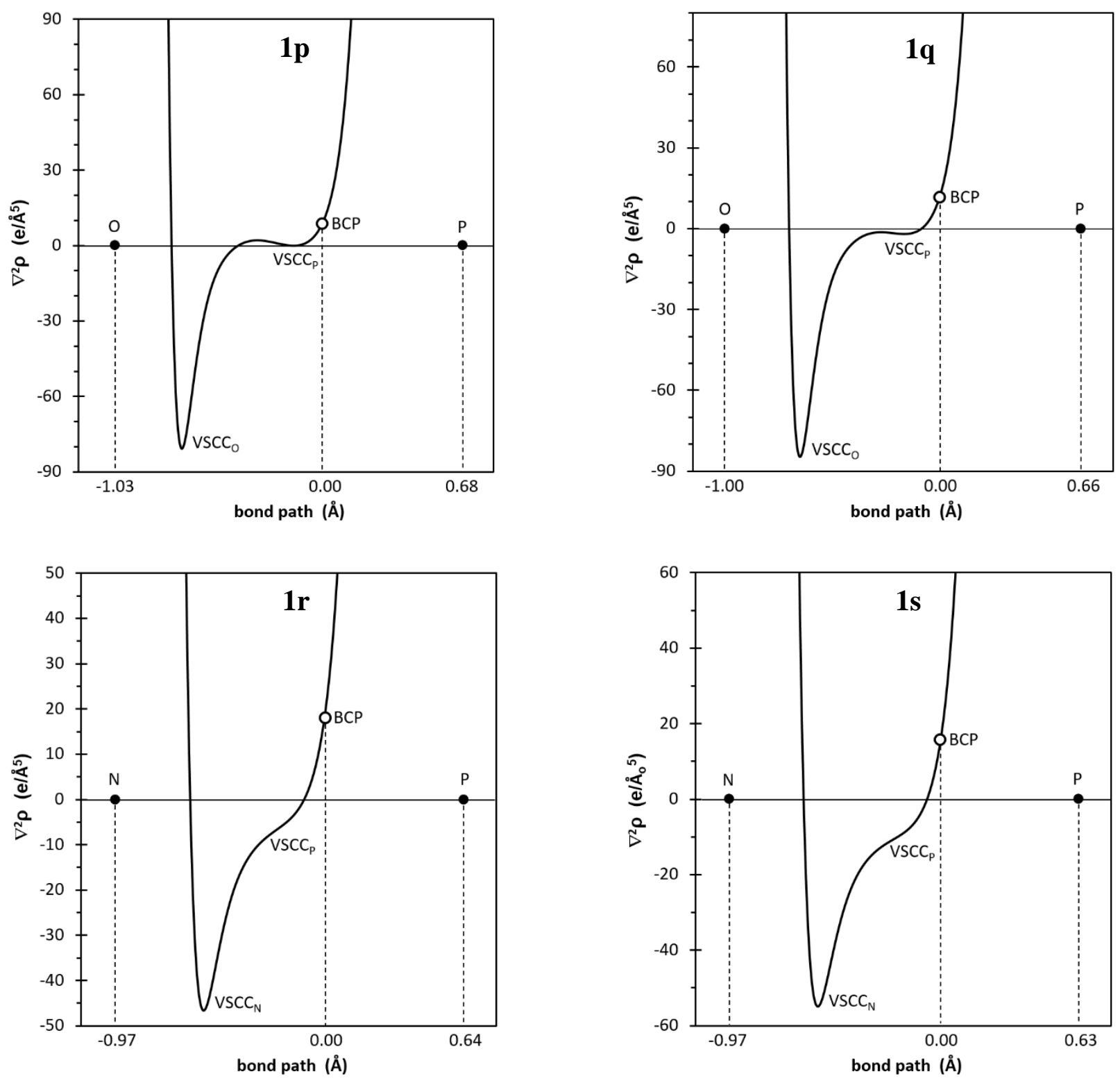

Figure S11. Computed (B3LYP-D3/def2-TZVPPecp//B3LYP-D3/def2-TZVPecp) variation of the Laplacian of electron density $\nabla^{2} \rho$ for adducts derived from nitric oxide 1p-s (L: $\underline{\mathrm{ON}}$, $\underline{\mathrm{ONP}}(\mathrm{Me}) \mathrm{W}(\mathrm{CO})_{5}$, $\underline{\mathrm{NO}}$ and $\underline{\mathrm{NOP}}(\mathrm{Me}) \mathrm{W}(\mathrm{CO})_{5}$, respectively) along the O-P or N-P bond paths. 


\section{Calculated structures}

Cartesian coordinates (in $\AA$ ) and ZPE (in hartrees) for minima and transition states were computed at COSMO(toluene)/B3LYP-D3/def2-TZVP(ecp), whereas electronic energies (in hartrees) are quoted at the COSMO(toluene)/DLPNO-CCSD(T)/def2-TZVPP(есp) level unless otherwise stated. In some cases, where explicitly indicated, geometries and ZPE were obtained at the CPCM(toluene)/PBEh-3c(ecp). Final electronic energies for open-shell species were computed at the LPNO-NCEPA1 level. Imaginary frequencies are obtained upon frequency calculation (at the optimization level).

\begin{tabular}{|c|c|c|c|c|c|c|c|}
\hline \multicolumn{3}{|c|}{ Me-P-W(CO)5: } & \multicolumn{5}{|c|}{$\begin{array}{l}\mathrm{E}=-1013.191277800 \mathrm{au}(\mathrm{COSMO}(\mathrm{tol}) / \mathrm{NCEPA} 1 / \text { def2-TZVPP(ecp)) } \\
\mathrm{E}=-1013.293181776 \mathrm{au}\end{array}$} \\
\hline$P$ & -0.068558 & 0.152939 & 2.334762 & $\mathrm{C}$ & 0.766828 & -2.855222 & 2.014461 \\
\hline W & -0.698134 & -2.004913 & 3.215457 & $\mathrm{O}$ & 1.590813 & -3.308597 & 1.367770 \\
\hline $\mathrm{C}$ & -1.223101 & -3.896601 & 3.946557 & $\mathrm{C}$ & 0.768618 & -1.809993 & 4.678870 \\
\hline $\mathrm{O}$ & -1.545716 & -4.919083 & 4.336775 & $\mathrm{O}$ & 1.584773 & -1.699644 & 5.468134 \\
\hline $\mathrm{C}$ & -2.105583 & -1.055239 & 4.399536 & $\mathrm{C}$ & -1.542615 & 1.238537 & 2.101639 \\
\hline $\mathrm{O}$ & -2.873391 & -0.488960 & 5.027274 & $\mathrm{H}$ & -2.504497 & 0.730692 & 2.054575 \\
\hline $\mathrm{C}$ & -2.155053 & -2.146994 & 1.745758 & $\mathrm{H}$ & -1.549586 & 1.907546 & 2.972999 \\
\hline $\mathrm{O}$ & -2.964937 & -2.182017 & 0.942118 & $\mathrm{H}$ & -1.405392 & 1.876304 & 1.226165 \\
\hline
\end{tabular}

\begin{tabular}{|c|c|c|c|c|c|c|c|}
\hline \multicolumn{3}{|c|}{ Me-P-W(CO) } & \multicolumn{5}{|c|}{$\begin{array}{l}\mathrm{E}=-1013.529611219 \text { au (optimized with CPCM(tol)/PBEh-3c(ecp)) } \\
\mathrm{ZPE}=0.08087095 \mathrm{au}\end{array}$} \\
\hline P & 0.628051 & -0.443366 & 0.378533 & $\mathrm{C}$ & 0.409261 & 1.343300 & 2.976065 \\
\hline $\mathrm{C}$ & 2.424660 & -0.067474 & 0.526784 & $\mathrm{O}$ & 1.056940 & 1.347029 & 3.911269 \\
\hline $\mathrm{H}$ & 2.693269 & 0.726895 & 1.220385 & $\mathrm{C}$ & 0.601335 & 2.704028 & 0.458883 \\
\hline $\mathrm{H}$ & 2.982043 & -0.973673 & 0.768213 & $\mathrm{O}$ & 1.353114 & 3.438580 & 0.022441 \\
\hline $\mathrm{H}$ & 2.756717 & 0.228681 & -0.475358 & $\mathrm{C}$ & -1.843584 & 1.304131 & -0.500286 \\
\hline $\mathrm{W}$ & -0.722589 & 1.326407 & 1.244232 & $\mathrm{O}$ & -2.460897 & 1.281921 & -1.454906 \\
\hline C & -1.952993 & 2.835861 & 2.004418 & $\mathrm{C}$ & -2.018688 & -0.102507 & 1.994507 \\
\hline $\mathrm{O}$ & -2.604901 & 3.666626 & 2.426180 & $\mathrm{O}$ & -2.732630 & -0.893225 & 2.393372 \\
\hline
\end{tabular}

$\mathrm{F}^{-}: \quad \mathrm{E}=-99.793173057$

$\mathrm{Cl}^{-}: \quad \mathrm{E}=-459.836732322$

$\mathrm{Br}^{-}: \quad \mathrm{E}=-2572.725658560$

$\mathrm{I}^{-}: \quad \mathrm{E}=-295.892994542$ 


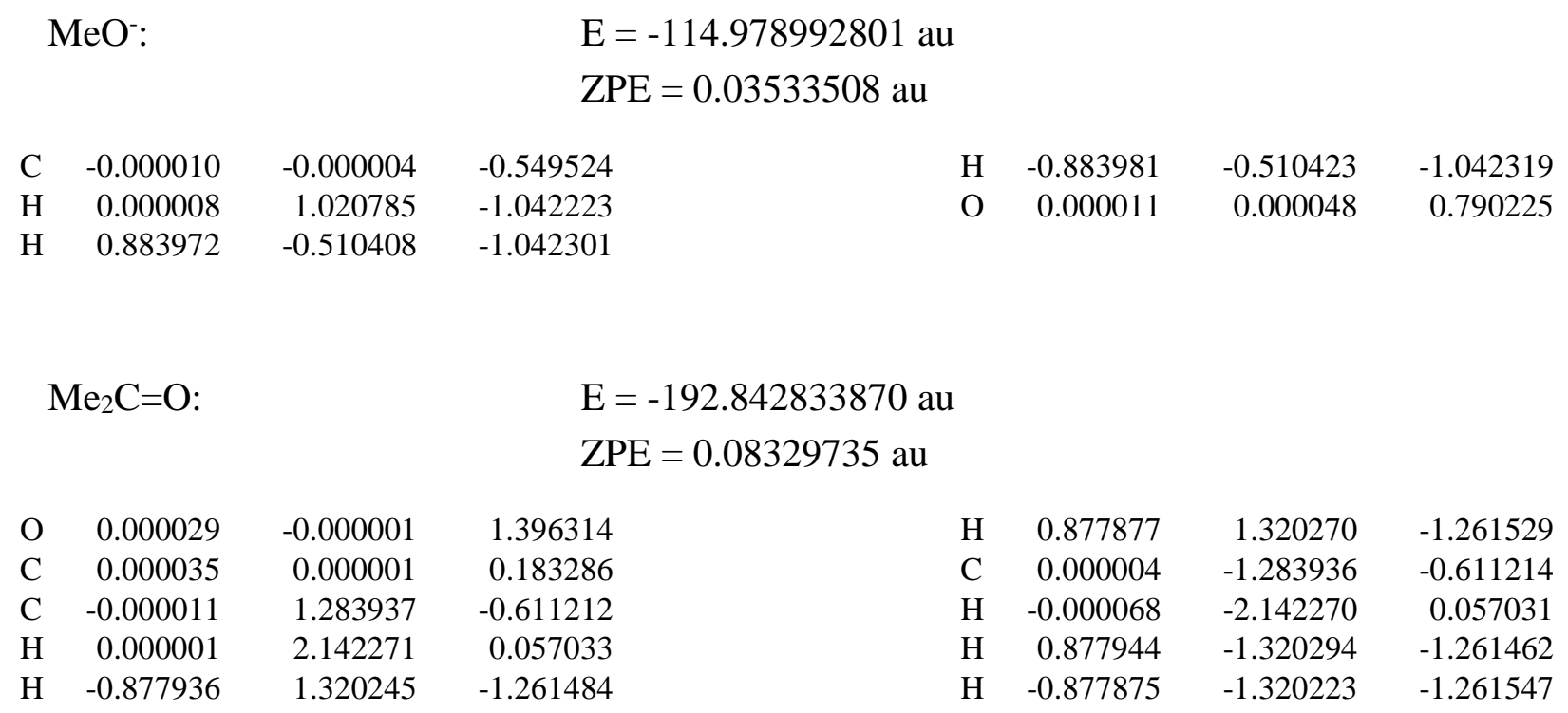

$\mathrm{E}=192.844078503$ au (optimized with CPCM(tol)/PBEh-3c(ecp))

$\mathrm{ZPE}=0.08597568 \mathrm{au}$

$\begin{array}{rrrrr}1.404102 & \mathrm{H} & 0.874764 & 1.309651 & -1.259629 \\ 0.196794 & \mathrm{C} & 0.000164 & -1.274580 & -0.607153 \\ -0.607154 & \mathrm{H} & 0.000860 & -2.150280 & 0.037789 \\ 0.037790 & \mathrm{H} & 0.874759 & -1.309649 & -1.259634 \\ -1.258848 & \mathrm{H} & -0.875010 & -1.310561 & -1.258840\end{array}$

$E=-188.332729448 \mathrm{au}$

$\mathrm{ZPE}=0.01009770 \mathrm{au}$

$\begin{array}{rrrr}\text { C } & -0.577251 & 0.040209 & -0.002364 \\ \text { O } & 0.256287 & -0.510673 & 0.586478\end{array}$

O $\quad-1.410748 \quad 0.591064 \quad-0.591290$

$E=-153.549910886 \mathrm{au}$

$\mathrm{ZPE}=0.05718969 \mathrm{au}$

C $\quad-2.010515$

0.559306

0.555139

0.000002

0.000002

H $\quad-2.546224$

0.344758

0.337558

0.919214

$-0.000007$

H $\quad-0.013309$

0.337549

$-0.919160$

0.919162

Oxetane:

$$
E=-192.793232795 \mathrm{au}
$$$$
\mathrm{ZPE}=0.08663339 \mathrm{au}
$$

$\begin{array}{lrrr}\mathrm{O} & -0.326816 & 0.694085 & -1.683145 \\ \mathrm{C} & 0.244477 & 1.890805 & -2.272625 \\ \mathrm{C} & 0.130184 & 1.302625 & -3.689127 \\ \mathrm{C} & -0.470395 & 0.073132 & -2.986882 \\ \mathrm{H} & -0.372179 & 2.770091 & -2.070914\end{array}$

$\begin{array}{rrrr}\mathrm{H} & 1.259963 & 2.069670 & -1.910985 \\ \mathrm{H} & -0.546797 & 1.831290 & -4.356946 \\ \mathrm{H} & 1.077354 & 1.129143 & -4.195647 \\ \mathrm{H} & -1.517313 & -0.141395 & -3.214996 \\ \mathrm{H} & 0.112172 & -0.848600 & -3.057719\end{array}$


THF:

$\begin{array}{lrrr}\mathrm{O} & -0.368674 & -1.453225 & -0.564882 \\ \mathrm{C} & -0.529848 & -0.702105 & -1.779439 \\ \mathrm{C} & -1.637714 & -1.395940 & -2.569601 \\ \mathrm{C} & -2.493494 & -1.999229 & -1.451333 \\ \mathrm{C} & -1.430473 & -2.410961 & -0.437684 \\ \mathrm{H} & -0.807610 & 0.326894 & -1.525821 \\ \mathrm{H} & 0.425291 & -0.681846 & -2.307965\end{array}$

H 0.425291
$E=-232.064176480 \mathrm{au}$

$\mathrm{ZPE}=0.11630071 \mathrm{au}$$$
\mathrm{ZPE}=0.11630071 \mathrm{au}
$$

\begin{tabular}{lrrr}
\multicolumn{2}{c}{$\mathrm{Me}_{3} \mathrm{NO}:$} & $\mathrm{E}=$ \\
& & & $\mathrm{ZPE}$ \\
$\mathrm{O}$ & 0.036226 & 0.050084 & 0.058750 \\
$\mathrm{~N}$ & 1.402750 & -0.007917 & -0.006605 \\
$\mathrm{C}$ & 1.964766 & 1.378328 & -0.002921 \\
$\mathrm{H}$ & 3.054012 & 1.356121 & -0.054690 \\
$\mathrm{H}$ & 1.546381 & 1.895552 & -0.861212 \\
$\mathrm{H}$ & 1.629948 & 1.856913 & 0.912557 \\
$\mathrm{C}$ & 1.815846 & -0.706606 & -1.262905
\end{tabular}

$E=-249.232485512 \mathrm{au}$

$\mathrm{ZPE}=0.12534256 \mathrm{au}$

\begin{tabular}{lrrr}
\multicolumn{2}{c}{$\mathrm{Me}_{3} \mathrm{PO}:$} & $\mathrm{E}=$ \\
& & & $\mathrm{ZPE}$ \\
$\mathrm{O}$ & -0.536841 & 0.932403 & -1.510337 \\
$\mathrm{P}$ & -1.497694 & 0.408831 & -2.523040 \\
$\mathrm{C}$ & -0.875666 & -1.030330 & -3.432623 \\
$\mathrm{H}$ & -1.601632 & -1.382321 & -4.166936 \\
$\mathrm{H}$ & 0.049205 & -0.756266 & -3.941523 \\
$\mathrm{H}$ & -0.660321 & -1.831797 & -2.724931 \\
$\mathrm{C}$ & -1.935882 & 1.623196 & -3.795201
\end{tabular}

DMSO:

\begin{tabular}{lrrr}
\multicolumn{2}{c}{ DMSO: } & & E $=$ \\
& & & ZPE \\
& & & \\
O & -0.183668 & 0.985204 & -1.534443 \\
S & -1.001241 & 0.720049 & -2.760745 \\
C & -0.412685 & -0.852361 & -3.441167 \\
H & -1.047960 & -1.147706 & -4.276480 \\
H & 0.604672 & -0.681967 & -3.789305
\end{tabular}

$\begin{array}{lrrr}\mathrm{H} & -2.637500 & 1.207616 & -4.519833 \\ \mathrm{H} & -2.385478 & 2.493136 & -3.314944 \\ \mathrm{H} & -1.028525 & 1.939617 & -4.311032 \\ \mathrm{C} & -3.077857 & -0.127007 & -1.814595 \\ \mathrm{H} & -3.752744 & -0.501510 & -2.585692 \\ \mathrm{H} & -2.891400 & -0.915440 & -1.084445 \\ \mathrm{H} & -3.544379 & 0.717184 & -1.305503\end{array}$

$\begin{array}{llll}\mathrm{H} & 2.902581 & -0.765041 & -1.335513 \\ \mathrm{H} & 1.375142 & -1.698762 & -1.236935 \\ \mathrm{H} & 1.401612 & -0.143130 & -2.093671 \\ \mathrm{C} & 1.930363 & -0.759215 & 1.174265 \\ \mathrm{H} & 3.019083 & -0.817144 & 1.142954 \\ \mathrm{H} & 1.595564 & -0.233584 & 2.063577 \\ \mathrm{H} & 1.488515 & -1.750821 & 1.146362\end{array}$

$\mathrm{E}=-535.615801678 \mathrm{au}$

$\mathrm{ZPE}=0.11676070 \mathrm{au}$

H $\quad-1.786339 \quad-2.396810 \quad 0.593906$

H $\quad-1.042885 \quad-3.412680 \quad-0.657932$ 


\begin{tabular}{|c|c|c|c|c|c|c|c|}
\hline & O: & & $\begin{array}{l}\mathrm{E}= \\
\mathrm{ZP}\end{array}$ & & & & \\
\hline $\mathrm{N}$ & 2.527248 & -2.277897 & -1.012754 & $\mathrm{O}$ & 0.654066 & -1.723054 & 0.205965 \\
\hline $\mathrm{N}$ & 1.615703 & -2.008253 & -0.420214 & & & & \\
\hline
\end{tabular}

\begin{tabular}{|c|c|c|c|c|c|c|c|}
\hline \multicolumn{3}{|c|}{ NO: } & \multicolumn{5}{|c|}{$\begin{array}{l}\mathrm{E}=-129.705829692 \mathrm{au}(\mathrm{COSMO}(\mathrm{tol}) / \mathrm{NCEPA} 1 / \text { def2-TZVPP) } \\
\mathrm{ZPE}=0.00450092 \mathrm{au}\end{array}$} \\
\hline $\mathrm{O}$ & -0.058490 & -0.085102 & -0.000056 & $\mathrm{~N}$ & 1.084276 & -0.019209 & 0.000056 \\
\hline \multicolumn{3}{|c|}{ s-cis-(NO) $)_{2}$} & \multicolumn{3}{|c|}{$E=-259.435518886$ au } & & \\
\hline $\mathrm{O}$ & 0.026735 & 0.096973 & -0.000029 & $\mathrm{~N}$ & 1.549672 & 2.040046 & -0.000036 \\
\hline $\mathrm{N}$ & 1.173106 & 0.102858 & 0.000036 & $\mathrm{O}$ & 0.488954 & 2.474936 & 0.000029 \\
\hline \multicolumn{3}{|c|}{ Cyclic-(NO) $)_{2}:$} & \multicolumn{3}{|c|}{$\begin{array}{l}E=-259.373905396 \mathrm{au} \\
Z P E=0.01406958 \mathrm{au}\end{array}$} & & \\
\hline $\mathrm{O}$ & 2.094657 & -1.117067 & -0.661316 & $\mathrm{~N}$ & 1.661447 & -2.692509 & 0.367561 \\
\hline $\mathrm{N}$ & 2.570198 & -1.838515 & 0.419916 & $\mathrm{O}$ & 1.037285 & -2.111031 & -0.721970 \\
\hline
\end{tabular}

1a:

$\begin{array}{lrrr}\mathrm{P} & 0.226943 & -0.653947 & 0.540862 \\ \mathrm{C} & 1.776389 & 0.105147 & -0.142637 \\ \mathrm{H} & 1.601183 & 1.037830 & -0.682769 \\ \mathrm{H} & 2.444821 & 0.310034 & 0.697510 \\ \mathrm{H} & 2.273373 & -0.604359 & -0.809182 \\ \mathrm{~W} & -1.048091 & 1.289950 & 1.798223 \\ \mathrm{C} & -2.052846 & 2.786266 & 2.692152 \\ \mathrm{O} & -2.626771 & 3.665079 & 3.186850 \\ \mathrm{C} & 0.771838 & 2.056248 & 2.344635\end{array}$

1b:

$\begin{array}{lrrr}\mathrm{P} & 0.288223 & -0.664391 & 0.616018 \\ \mathrm{C} & 1.804695 & 0.117036 & -0.126683 \\ \mathrm{H} & 1.592189 & 1.012904 & -0.711290 \\ \mathrm{H} & 2.461128 & 0.389873 & 0.704296 \\ \mathrm{H} & 2.326283 & -0.605854 & -0.756847\end{array}$

$$
\begin{aligned}
& E=-1473.200039572 \mathrm{au} \\
& Z P E=0.07995192 \mathrm{au}
\end{aligned}
$$

$\begin{array}{llll}\text { O } & 1.810018 & 2.462478 & 2.638807\end{array}$

$\begin{array}{llll}\text { C } & -0.888677 & 2.228900 & -0.021762\end{array}$

$\begin{array}{llll}\text { O } & -0.746642 & 2.703153 & -1.060121\end{array}$

$\begin{array}{llll}\text { C } & -2.779601 & 0.360620 & 1.181518\end{array}$

$\begin{array}{llll}\text { O } & -3.734195 & -0.179884 & 0.835820\end{array}$

$\begin{array}{llll}\text { C } & -1.028181 & 0.112314 & 3.478041\end{array}$

$\begin{array}{llll}\mathrm{O} & -0.992805 & -0.577492 & 4.400210\end{array}$

$\begin{array}{llll}\text { F } & -0.573376 & -0.780434 & -0.927653\end{array}$ 


$\begin{array}{lrrrrrrr}\mathrm{C} & -0.875419 & 2.241721 & -0.009637 & \mathrm{C} & -1.031025 & 0.118083 & 3.493551 \\ \mathrm{O} & -0.741166 & 2.734804 & -1.038363 & \mathrm{O} & -1.009846 & -0.560214 & 4.423657 \\ \mathrm{C} & -2.780697 & 0.373647 & 1.196688 & \mathrm{Cl} & -0.741172 & -1.008942 & -1.271121 \\ \mathrm{O} & -3.751029 & -0.142245 & 0.863982 & & & \end{array}$

1c:

$\begin{array}{lrrr}\text { P } & 0.007190 & -0.020926 & 0.015389 \\ \text { C } & 1.869329 & -0.001548 & 0.006754 \\ \text { H } & 2.298242 & 1.000729 & -0.006684 \\ \text { H } & 2.190377 & -0.504647 & 0.923551 \\ \text { H } & 2.247837 & -0.564920 & -0.847763 \\ \text { W } & -0.729956 & 1.646617 & 1.916223 \\ \text { C } & -1.272384 & 2.908141 & 3.383370 \\ \text { O } & -1.565815 & 3.647439 & 4.227305 \\ \text { C } & 0.843647 & 0.962351 & 3.036250\end{array}$

$E=-3586.077264592 \mathrm{au}$

$$
\mathrm{ZPE}=0.07948766 \mathrm{au}
$$

$\begin{array}{lrrr}\mathrm{O} & 1.728458 & 0.570401 & 3.661577 \\ \mathrm{C} & 0.524162 & 2.994725 & 0.993917 \\ \mathrm{O} & 1.249996 & 3.701027 & 0.452807 \\ \mathrm{C} & -2.302230 & 2.251646 & 0.721811 \\ \mathrm{O} & -3.184435 & 2.581412 & 0.065906 \\ \mathrm{C} & -1.900255 & 0.127772 & 2.648827 \\ \mathrm{O} & -2.544634 & -0.742390 & 3.039280 \\ \mathrm{Br} & -0.261462 & 1.138678 & -2.023073\end{array}$

1d:

$\begin{array}{lrrr}\text { P } & 0.017816 & -0.037929 & 0.028345 \\ \text { C } & 1.883172 & 0.012993 & 0.007799 \\ \text { H } & 2.297921 & 1.020287 & -0.029397 \\ \text { H } & 2.214630 & -0.463649 & 0.935088 \\ \text { H } & 2.268381 & -0.563609 & -0.834462 \\ \text { W } & -0.730529 & 1.647118 & 1.909786 \\ \text { C } & -1.265468 & 2.897052 & 3.388832 \\ \text { O } & -1.553507 & 3.627734 & 4.241851 \\ \text { C } & 0.835901 & 0.955517 & 3.036567\end{array}$

$E=-1309.262363546 \mathrm{au}$

$\mathrm{ZPE}=0.07936438 \mathrm{au}$ 1e:

$\begin{array}{lr}\text { P } & 0.157866 \\ \text { C } & 2.022386 \\ \text { H } & 2.397348 \\ \text { H } & 2.366191 \\ \text { H } & 2.451576 \\ \text { W } & -0.698309 \\ \text { C } & -1.353063 \\ \text { O } & -1.714336 \\ \text { C } & 0.912712 \\ \text { O } & 1.826292 \\ \text { C } & 0.468773\end{array}$

1f:

$\begin{array}{lrrr}\mathrm{O} & 1.713772 & 0.563371 & 3.670943 \\ \mathrm{C} & 0.530594 & 2.996338 & 0.998375 \\ \mathrm{O} & 1.260063 & 3.703798 & 0.463908 \\ \mathrm{C} & -2.306474 & 2.276099 & 0.732361 \\ \mathrm{O} & -3.193452 & 2.623487 & 0.092528 \\ \mathrm{C} & -1.912111 & 0.130238 & 2.630095 \\ \mathrm{O} & -2.563001 & -0.737382 & 3.014166 \\ \mathrm{I} & -0.319749 & 1.142300 & -2.233316\end{array}$

$$
\begin{aligned}
& E=-1128.408989084 \mathrm{au} \\
& Z P E=0.12000074 \mathrm{au}
\end{aligned}
$$
0.034550
0.075642
0.039719
$-0.758861$
3.249829
3.051999

O $\quad 1.149462$

3.660973

0.249093

C $\quad-2.258618$

1.997761

0.609464

O -3.122775

2.177739

$-0.127578$

$\begin{array}{ll}-0.399907 & 1.002748\end{array}$

C -1.755586

0.076672

2.720366

$-0.823050$

3.150665

O -2.333432

0.872033

$-1.383313$

C -0.034360

0.168670

$-2.605637$

H $\quad-0.599018$

$-0.771089$

$-2.572832$

H $\quad-0.446615$

0.806628

$-3.391311$

H $\quad 1.006574$

$-0.067698$

$-2.861637$

$$
\begin{aligned}
& E=-1206.158410927 \mathrm{au} \\
& Z P E=0.16473994 \mathrm{au}
\end{aligned}
$$

$-0.160365$

C $\quad 2.746644$

0.953521

0.088821 


$\begin{array}{lrrr}\mathrm{H} & 2.709542 & 2.025930 & -0.099660 \\ \mathrm{H} & 3.007300 & 0.799448 & 1.139257 \\ \mathrm{H} & 3.524113 & 0.496260 & -0.524410 \\ \mathrm{~W} & -0.704778 & 1.408581 & 1.166934 \\ \mathrm{C} & -2.094731 & 2.355409 & 2.290826 \\ \mathrm{O} & -2.883978 & 2.903616 & 2.924805 \\ \mathrm{C} & 0.231681 & 0.625114 & 2.835854 \\ \mathrm{O} & 0.777498 & 0.187399 & 3.743161 \\ \mathrm{C} & 0.522347 & 3.062132 & 1.262532 \\ \mathrm{O} & 1.199527 & 3.985687 & 1.307612 \\ \mathrm{C} & -1.466904 & 2.126770 & -0.589475 \\ \mathrm{O} & -1.825711 & 2.483318 & -1.623821\end{array}$

$\begin{array}{lrrr}\mathrm{C} & -1.913310 & -0.254290 & 1.051572 \\ \mathrm{O} & -2.592245 & -1.177433 & 0.982407 \\ \mathrm{O} & 0.982887 & 0.728231 & -1.869251 \\ \mathrm{C} & 0.170756 & 0.326868 & -2.740143 \\ \mathrm{C} & -0.803407 & -0.737634 & -2.431137 \\ \mathrm{H} & -0.279744 & -1.557532 & -1.924821 \\ \mathrm{H} & -1.535034 & -0.362925 & -1.709675 \\ \mathrm{H} & -1.320285 & -1.087754 & -3.320246 \\ \mathrm{C} & 0.240871 & 0.946216 & -4.083845 \\ \mathrm{H} & 0.451986 & 0.170314 & -4.826383 \\ \mathrm{H} & -0.736225 & 1.365554 & -4.338481 \\ \mathrm{H} & 1.002182 & 1.720874 & -4.122543\end{array}$

1f:

$\mathrm{E}=-1206.393665059$ au (optimized with CPCM(tol)/PBEh-3c(ecp)) $\mathrm{ZPE}=0.171189510 \mathrm{au}$

$\begin{array}{lrrr}\mathrm{P} & 1.052598 & 0.069065 & -0.153571 \\ \mathrm{C} & 2.715188 & 0.803877 & 0.146561 \\ \mathrm{H} & 2.762073 & 1.883696 & 0.013738 \\ \mathrm{H} & 2.955728 & 0.589044 & 1.190209 \\ \mathrm{H} & 3.482204 & 0.331727 & -0.467423 \\ \mathrm{~W} & -0.704318 & 1.420051 & 1.167951 \\ \mathrm{C} & -2.065495 & 2.395179 & 2.284875 \\ \mathrm{O} & -2.839063 & 2.958208 & 2.919021 \\ \mathrm{C} & 0.269656 & 0.776836 & 2.868035 \\ \mathrm{O} & 0.818701 & 0.428227 & 3.806211 \\ \mathrm{C} & 0.495603 & 3.088244 & 1.124331 \\ \mathrm{O} & 1.151325 & 4.023168 & 1.097138 \\ \mathrm{C} & -1.574903 & 2.056957 & -0.563635\end{array}$

$\begin{array}{lrrr}\mathrm{O} & -2.013864 & 2.390469 & -1.569117 \\ \mathrm{C} & -1.880632 & -0.264190 & 1.180787 \\ \mathrm{O} & -2.539285 & -1.198384 & 1.182358 \\ \mathrm{O} & 0.950944 & 0.770515 & -1.849023 \\ \mathrm{C} & 0.192692 & 0.367047 & -2.751151 \\ \mathrm{C} & -0.731057 & -0.767446 & -2.538092 \\ \mathrm{H} & -0.186176 & -1.608211 & -2.097450 \\ \mathrm{H} & -1.501750 & -0.498884 & -1.810863 \\ \mathrm{H} & -1.216277 & -1.072402 & -3.460828 \\ \mathrm{C} & 0.242288 & 1.049641 & -4.063672 \\ \mathrm{H} & 0.500076 & 0.321342 & -4.836093 \\ \mathrm{H} & -0.753184 & 1.422361 & -4.313654 \\ \mathrm{H} & 0.955140 & 1.869139 & -4.077117\end{array}$

1g:

$E=-1201.628796905 \mathrm{au}$

$\mathrm{ZPE}=0.08974635 \mathrm{au}$

$\begin{array}{lrrr}\mathrm{P} & 0.059994 & 0.123203 & -0.196662 \\ \mathrm{C} & -0.453487 & 0.280389 & 1.566487 \\ \mathrm{H} & 0.133990 & 0.967531 & 2.172802 \\ \mathrm{H} & -1.490348 & 0.641173 & 1.537291 \\ \mathrm{H} & -0.485322 & -0.705970 & 2.033980 \\ \mathrm{~W} & 1.512597 & 1.927648 & -0.869901 \\ \mathrm{C} & 2.825175 & 3.443577 & -1.475521 \\ \mathrm{O} & 3.543585 & 4.275895 & -1.780156 \\ \mathrm{C} & 0.360364 & 3.233873 & 0.254727 \\ \mathrm{O} & -0.289954 & 3.913198 & 0.902191\end{array}$

$\begin{array}{rrrr}\mathrm{C} & 2.637214 & 1.588999 & 0.839321 \\ \mathrm{O} & 3.221421 & 1.387159 & 1.798391 \\ \mathrm{C} & 2.586857 & 0.523153 & -1.947896 \\ \mathrm{O} & 3.153714 & -0.279890 & -2.530483 \\ \mathrm{C} & 0.349805 & 2.222397 & -2.570060 \\ \mathrm{O} & -0.284035 & 2.366422 & -3.507518 \\ \mathrm{O} & 2.320120 & -2.041218 & 0.408618 \\ \mathrm{C} & 2.017082 & -2.689448 & -0.506673 \\ \mathrm{O} & 1.718130 & -3.335924 & -1.419251\end{array}$

1h:

$$
\begin{aligned}
& E=-1166.862099758 \mathrm{au} \\
& Z P E=0.13855853 \mathrm{au}
\end{aligned}
$$

$\begin{array}{rrrr}\mathrm{P} & -0.371269 & 0.047868 & -0.095872 \\ \mathrm{C} & 1.406151 & -0.413920 & 0.090123 \\ \mathrm{H} & 2.071829 & 0.438981 & 0.221730 \\ \mathrm{H} & 1.456221 & -1.027314 & 0.994630 \\ \mathrm{H} & 1.750161 & -1.029425 & -0.743261\end{array}$

$\begin{array}{lrrr}\text { W } & -0.930005 & 1.895368 & 1.557383 \\ \mathrm{C} & -1.375284 & 3.339316 & 2.925793 \\ \mathrm{O} & -1.609344 & 4.160230 & 3.695897 \\ \mathrm{C} & 0.239013 & 0.909729 & 2.945009 \\ \mathrm{O} & 0.898500 & 0.354624 & 3.699978\end{array}$ 


$\begin{array}{lrrr}\mathrm{C} & 0.737178 & 2.888887 & 0.870363 \\ \mathrm{O} & 1.675323 & 3.398826 & 0.452020 \\ \mathrm{C} & -2.081623 & 2.848939 & 0.144895 \\ \mathrm{O} & -2.719248 & 3.379543 & -0.650496 \\ \mathrm{C} & -2.568004 & 0.769879 & 2.113282 \\ \mathrm{O} & -3.475333 & 0.131770 & 2.401334 \\ \mathrm{O} & 0.048488 & 1.088327 & -1.717262\end{array}$

C $\quad 0.018249$

C $\quad-1.058942$

$\mathrm{H} \quad 0.815373$

H $\quad-0.149329$

H -1.075161

H $\quad-1.997237$
0.387715

1.303423

0.710607

$-0.676822$

2.312397

0.892647
$-3.010919$

$-2.653404$

$-3.666024$

$-2.909113$

$-3.039872$

$-2.304151$

$\begin{array}{lrrr}\text { 1i: } & & \mathrm{E}= \\ & & & \mathrm{ZP} \\ & & & \\ \mathrm{P} & -0.360247 & -0.076963 & -0.064893 \\ \mathrm{C} & 1.465417 & -0.374270 & -0.155520 \\ \mathrm{H} & 2.077036 & 0.528669 & -0.166178 \\ \mathrm{H} & 1.707350 & -0.922949 & 0.759306 \\ \mathrm{H} & 1.734943 & -1.016093 & -0.996847 \\ \mathrm{~W} & -0.787978 & 1.827073 & 1.609050 \\ \mathrm{C} & -1.170682 & 3.270674 & 2.986059 \\ \mathrm{O} & -1.375202 & 4.096111 & 3.761591 \\ \mathrm{C} & 0.475702 & 0.837177 & 2.903936 \\ \mathrm{O} & 1.193516 & 0.275416 & 3.599838 \\ \mathrm{C} & 0.823448 & 2.865944 & 0.887781 \\ \mathrm{O} & 1.736241 & 3.427756 & 0.469717 \\ \mathrm{C} & -2.009304 & 2.727799 & 0.220977\end{array}$

$\mathrm{E}=-1206.113275749 \mathrm{au}$

$\mathrm{ZPE}=0.16847032 \mathrm{au}$
$1 \mathrm{j}$ :

$\begin{array}{lrrr}\text { P } & 0.857549 & -1.261225 & 0.786496 \\ \mathrm{C} & 2.343239 & -0.943992 & -0.261614 \\ \mathrm{H} & 2.288228 & -0.051801 & -0.885684 \\ \mathrm{H} & 3.164571 & -0.801793 & 0.446640 \\ \mathrm{H} & 2.580764 & -1.814861 & -0.874808 \\ \mathrm{~W} & 0.073367 & 0.957072 & 1.833428 \\ \mathrm{C} & -0.432410 & 2.671623 & 2.798178 \\ \mathrm{O} & -0.710439 & 3.650439 & 3.335440 \\ \mathrm{C} & 2.008524 & 1.106564 & 2.542007 \\ \mathrm{O} & 3.086188 & 1.184498 & 2.923780 \\ \mathrm{C} & 0.638045 & 1.962738 & 0.136597 \\ \mathrm{O} & 0.964189 & 2.489598 & -0.831324 \\ \mathrm{C} & -1.839938 & 0.774086 & 1.115863 \\ \mathrm{O} & -2.909728 & 0.662486 & 0.706957 \\ \mathrm{C} & -0.462735 & -0.190757 & 3.465035\end{array}$

$$
E=-1245.382490431 \mathrm{au}
$$$$
\mathrm{ZPE}=0.19842980 \mathrm{au}
$$

$\begin{array}{lrrr}\mathrm{O} & -2.669236 & 3.212972 & -0.584489 \\ \mathrm{C} & -2.388430 & 0.702707 & 2.287800 \\ \mathrm{O} & -3.272590 & 0.075340 & 2.655747 \\ \mathrm{O} & -0.507288 & 0.773120 & -1.717031 \\ \mathrm{C} & 0.268459 & 1.916385 & -2.289197 \\ \mathrm{C} & 0.102871 & 1.313307 & -3.687272 \\ \mathrm{C} & -0.464212 & 0.056658 & -3.017684 \\ \mathrm{H} & -0.241998 & 2.846236 & -2.064316 \\ \mathrm{H} & 1.277900 & 1.909630 & -1.889787 \\ \mathrm{H} & -0.621895 & 1.841190 & -4.300774 \\ \mathrm{H} & 1.025836 & 1.167677 & -4.240730 \\ \mathrm{H} & -1.465942 & -0.265378 & -3.284703 \\ \mathrm{H} & 0.216919 & -0.787978 & -2.947491\end{array}$

1k:

$\begin{array}{lrrr}\mathrm{P} & -0.442672 & -0.040220 & -0.023800 \\ \mathrm{C} & 1.389280 & -0.158767 & -0.298177 \\ \mathrm{H} & 1.861754 & 0.819655 & -0.394128 \\ \mathrm{H} & 1.807017 & -0.654815 & 0.581457 \\ \mathrm{H} & 1.635860 & -0.766946 & -1.170682\end{array}$

$\begin{array}{lrrr}\mathrm{O} & -0.765748 & -0.845899 & 4.354630 \\ \mathrm{O} & -0.259492 & -1.620891 & -0.709042 \\ \mathrm{C} & -0.530255 & -0.687014 & -1.812039 \\ \mathrm{C} & -1.627815 & -1.387001 & -2.582367 \\ \mathrm{C} & -2.479789 & -2.011143 & -1.468486 \\ \mathrm{C} & -1.461386 & -2.432382 & -0.417888 \\ \mathrm{H} & -0.851462 & 0.258785 & -1.380802 \\ \mathrm{H} & 0.403913 & -0.558724 & -2.349200 \\ \mathrm{H} & -2.191501 & -0.687318 & -3.197606 \\ \mathrm{H} & -1.204718 & -2.156700 & -3.229436 \\ \mathrm{H} & -3.162686 & -1.270336 & -1.055501 \\ \mathrm{H} & -3.063482 & -2.861702 & -1.815663 \\ \mathrm{H} & -1.766610 & -2.215247 & 0.602945 \\ \mathrm{H} & -1.143595 & -3.468881 & -0.498296\end{array}$

$\begin{array}{llll}\mathrm{O} & -0.765748 & -0.845899 & 4.354630\end{array}$

$-1.620891 \quad-0.709042$

$-0.687014-1.812039$

$-1.387001 \quad-2.582367$

$-1.380802$

$-2.349200$

$-3.197606$

$-1.055501$

$-1.815663$

$-0.498296$

$$
\begin{aligned}
& E=-1262.586655244 \mathrm{au} \\
& Z P E=0.20673588 \mathrm{au}
\end{aligned}
$$

$\begin{array}{lrrrr}\text { ZPE }=0.20673588 \text { au } & & & \\ & \text { W } & -0.865234 & 1.654126 & 1.905077 \\ 3800 & \text { C } & -1.209657 & 2.994389 & 3.378662 \\ 177 & \text { O } & -1.400146 & 3.773977 & 4.207763 \\ 428 & \text { C } & 0.804569 & 0.914290 & 2.861235 \\ 457 & \text { O } & 1.738686 & 0.484455 & 3.371330\end{array}$




$\begin{array}{rrrr}\mathrm{C} & 0.317869 & 2.959215 & 0.842404 \\ \mathrm{O} & 0.983251 & 3.654345 & 0.215662 \\ \mathrm{C} & -2.492643 & 2.277185 & 0.827401 \\ \mathrm{O} & -3.388130 & 2.591658 & 0.174727 \\ \mathrm{C} & -2.022203 & 0.220377 & 2.825648 \\ \mathrm{O} & -2.668204 & -0.593162 & 3.314434 \\ \mathrm{O} & -0.799519 & 1.044815 & -1.378377 \\ \mathrm{~N} & -1.456565 & 0.479959 & -2.544771 \\ \mathrm{C} & -0.633194 & -0.613106 & -3.128662 \\ \mathrm{H} & -1.143373 & -0.997897 & -4.008703\end{array}$

$\begin{array}{lrrr}\mathrm{H} & 0.336232 & -0.200831 & -3.390479 \\ \mathrm{H} & -0.533317 & -1.388078 & -2.372569 \\ \mathrm{C} & -1.557276 & 1.637097 & -3.471595 \\ \mathrm{H} & -2.066131 & 1.316091 & -4.377717 \\ \mathrm{H} & -2.118565 & 2.417069 & -2.966553 \\ \mathrm{H} & -0.550672 & 1.979435 & -3.690499 \\ \mathrm{C} & -2.809659 & -0.010033 & -2.153321 \\ \mathrm{H} & -3.304925 & -0.400109 & -3.039441 \\ \mathrm{H} & -2.672493 & -0.789063 & -1.407545 \\ \mathrm{H} & -3.358529 & 0.826953 & -1.733318\end{array}$

\section{1:}

$\mathrm{E}=-1548.946353795 \mathrm{au}$

$\mathrm{ZPE}=0.19763014 \mathrm{au}$

$\begin{array}{lrrr}\mathrm{P} & -0.130272 & 0.025941 & -0.069620 \\ \mathrm{C} & 1.690762 & 0.170473 & -0.332957 \\ \mathrm{H} & 2.027989 & 1.202403 & -0.434694 \\ \mathrm{H} & 2.171300 & -0.263700 & 0.547721 \\ \mathrm{H} & 2.005225 & -0.402452 & -1.207418 \\ \mathrm{~W} & -0.849717 & 1.634025 & 1.828539 \\ \mathrm{C} & -1.404292 & 2.843459 & 3.357938 \\ \mathrm{O} & -1.709790 & 3.539550 & 4.224773 \\ \mathrm{C} & 0.589801 & 0.698435 & 2.976724 \\ \mathrm{O} & 1.405067 & 0.163841 & 3.581699 \\ \mathrm{C} & 0.556823 & 2.959668 & 1.123908 \\ \mathrm{O} & 1.340332 & 3.672957 & 0.682170 \\ \mathrm{C} & -2.199142 & 2.474049 & 0.542739 \\ \mathrm{O} & -2.933531 & 2.912036 & -0.230756 \\ \mathrm{C} & -2.240506 & 0.206221 & 2.336634\end{array}$

$\begin{array}{llll}\text { O } & -3.019860 & -0.604816 & 2.570409\end{array}$

$\begin{array}{llll}\mathrm{O} & -0.554265 & 0.964576 & -1.586911\end{array}$

$\begin{array}{llll}\mathrm{P} & -1.565789 & 0.364960 & -2.595023\end{array}$

$\begin{array}{llll}\text { C } & -0.870558 & -1.057338 & -3.438457\end{array}$

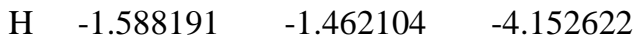

H $\quad 0.037553 \quad-0.757374 \quad-3.961273$

$\begin{array}{llll}\mathrm{H} & -0.626154 & -1.815692 & -2.694192\end{array}$

$\begin{array}{llll}\text { C } & -1.922878 & 1.653375 & -3.783425\end{array}$

$\mathrm{H} \quad-2.633375 \quad 1.290935 \quad-4.526895$

$\begin{array}{llll}\mathrm{H} & -2.348292 & 2.507737 & -3.256602\end{array}$

$\mathrm{H} \quad-0.999471 \quad 1.956314 \quad-4.276592$

$\begin{array}{llll}\text { C } & -3.095851 & -0.140109 & -1.809051\end{array}$

$\mathrm{H} \quad-3.765426 \quad-0.567062 \quad-2.556682$

$\mathrm{H} \quad-2.875914 \quad-0.886279 \quad-1.045789$

H $\quad-3.568103 \quad 0.725369 \quad-1.347120$

1m:

$$
\begin{aligned}
& E=-1565.837405543 \mathrm{au} \\
& Z P E=0.16005529 \mathrm{au}
\end{aligned}
$$

$\begin{array}{lrrr}\mathrm{P} & -0.205091 & -0.039521 & -0.013473 \\ \mathrm{C} & 1.632196 & -0.243588 & 0.000985 \\ \mathrm{H} & 2.167364 & 0.702623 & 0.085078 \\ \mathrm{H} & 1.868192 & -0.850613 & 0.879101 \\ \mathrm{H} & 1.980340 & -0.782172 & -0.882429 \\ \mathrm{~W} & -0.886459 & 1.558752 & 1.885574 \\ \mathrm{C} & -1.415438 & 2.794083 & 3.406126 \\ \mathrm{O} & -1.701847 & 3.503096 & 4.267817 \\ \mathrm{C} & 0.520850 & 0.620596 & 3.068314 \\ \mathrm{O} & 1.313781 & 0.086243 & 3.701895 \\ \mathrm{C} & 0.558496 & 2.845270 & 1.178249 \\ \mathrm{O} & 1.369731 & 3.529708 & 0.743341 \\ \mathrm{C} & -2.241986 & 2.413176 & 0.608262\end{array}$

$\begin{array}{lrrr}\mathrm{O} & -2.988545 & 2.866102 & -0.142503 \\ \mathrm{C} & -2.302050 & 0.166139 & 2.428653 \\ \mathrm{O} & -3.093511 & -0.620200 & 2.698817 \\ \mathrm{O} & -0.165738 & 1.126216 & -1.468377 \\ \mathrm{~S} & -0.844892 & 0.783413 & -2.833290 \\ \mathrm{C} & -0.212392 & -0.826721 & -3.315639 \\ \mathrm{H} & -0.763118 & -1.139932 & -4.202008 \\ \mathrm{H} & 0.841082 & -0.697632 & -3.554567 \\ \mathrm{H} & -0.351604 & -1.522888 & -2.489606 \\ \mathrm{C} & -2.539152 & 0.345665 & -2.436516 \\ \mathrm{H} & -3.015994 & 0.032268 & -3.364823 \\ \mathrm{H} & -2.540108 & -0.452172 & -1.695409 \\ \mathrm{H} & -3.017049 & 1.243867 & -2.051836\end{array}$

1n:

$$
\begin{aligned}
& E=-1163.347482114 \mathrm{au} \\
& \mathrm{ZPE}=0.08487163 \mathrm{au}
\end{aligned}
$$

$\begin{array}{lrrr}\mathrm{P} & -0.003688 & 0.003505 & -0.182606 \\ \mathrm{C} & 1.773097 & 0.071499 & 0.038310\end{array}$

$\mathrm{H} \quad 1.990233$

0.580405

0.980750

C 1.773097

0.071499

0.038310

H 2.182298

$-0.936915$

0.028126 


\begin{tabular}{|c|c|c|c|c|c|c|c|}
\hline $\mathrm{H}$ & 2.200265 & 0.683508 & -0.760100 & $\mathrm{O}$ & -0.799603 & -2.042249 & -3.606487 \\
\hline $\mathrm{O}$ & -0.479328 & 1.503564 & -0.161714 & $\mathrm{C}$ & 0.072522 & -3.099709 & -0.034615 \\
\hline $\mathrm{O}$ & 0.447634 & 2.533631 & 0.009346 & $\mathrm{O}$ & 0.957012 & -3.781523 & 0.206468 \\
\hline W & -1.501088 & -1.841379 & -0.470164 & $\mathrm{C}$ & -2.055711 & -1.784448 & 1.537390 \\
\hline C & -2.741863 & -3.475190 & -0.711085 & $\mathrm{O}$ & -2.372511 & -1.764488 & 2.630548 \\
\hline $\mathrm{O}$ & -3.422940 & -4.379764 & -0.843043 & C & -3.051325 & -0.520412 & -0.901517 \\
\hline C & -1.041840 & -1.961652 & -2.496556 & $\mathrm{O}$ & -3.898732 & 0.201788 & -1.137629 \\
\hline
\end{tabular}

10:

$$
\mathrm{E}=-1197.707526743 \mathrm{au}
$$$$
\mathrm{ZPE}=0.08960548 \mathrm{au}
$$

$\begin{array}{lrrr}\mathrm{P} & 0.164289 & 0.569886 & -0.377588 \\ \mathrm{C} & 0.177564 & 0.371501 & 1.457454 \\ \mathrm{H} & 1.156110 & 0.036629 & 1.805804 \\ \mathrm{H} & 0.040496 & 1.388009 & 1.850647 \\ \mathrm{H} & -0.611602 & -0.253180 & 1.874550 \\ \mathrm{~W} & -2.051304 & 0.213656 & -1.276298 \\ \mathrm{C} & -3.942913 & -0.105238 & -2.111784 \\ \mathrm{O} & -4.982397 & -0.290505 & -2.544860 \\ \mathrm{C} & -2.199291 & -1.488345 & -0.116307 \\ \mathrm{O} & -2.236364 & -2.407576 & 0.562621\end{array}$

$\begin{array}{lrrr}\mathrm{C} & -2.840352 & 1.260322 & 0.327064 \\ \mathrm{O} & -3.234821 & 1.825444 & 1.237895 \\ \mathrm{C} & -1.820127 & 1.947600 & -2.402783 \\ \mathrm{O} & -1.678786 & 2.893761 & -3.024506 \\ \mathrm{C} & -1.166874 & -0.830109 & -2.841532 \\ \mathrm{O} & -0.664939 & -1.393797 & -3.696978 \\ \mathrm{O} & 0.964891 & -2.462976 & -0.193816 \\ \mathrm{~N} & 0.872006 & -2.890536 & 0.906892 \\ \mathrm{~N} & 0.779965 & -3.286837 & 1.949439\end{array}$

1p:

$\mathrm{E}=-1142.881145694 \mathrm{au}(\mathrm{COSMO}(\mathrm{tol}) / \mathrm{NCEPA} 1 /$ def2-TZVPP(еср)) $\mathrm{ZPE}=0.08450017 \mathrm{au}$

$\begin{array}{lrrr}\mathrm{P} & -0.004442 & 0.026337 & 0.356612 \\ \mathrm{C} & 1.753040 & -0.007435 & -0.109036 \\ \mathrm{H} & 2.281632 & 0.856137 & 0.296655 \\ \mathrm{H} & 2.181071 & -0.922466 & 0.301522 \\ \mathrm{H} & 1.862948 & -0.016212 & -1.196173 \\ \mathrm{~N} & 0.279320 & 2.420424 & -0.821612 \\ \mathrm{O} & -0.479950 & 1.528851 & -0.314378 \\ \mathrm{~W} & -1.529130 & -1.823376 & -0.245398 \\ \mathrm{C} & -2.639108 & -3.390526 & -0.942156\end{array}$

$\begin{array}{lrrr}\mathrm{O} & -3.243843 & -4.267967 & -1.359767 \\ \mathrm{C} & -0.781854 & -1.559829 & -2.155193 \\ \mathrm{O} & -0.352084 & -1.400672 & -3.201511 \\ \mathrm{C} & 0.014979 & -3.112463 & 0.201172 \\ \mathrm{O} & 0.882575 & -3.815272 & 0.451226 \\ \mathrm{C} & -2.288894 & -2.073384 & 1.674323 \\ \mathrm{O} & -2.718670 & -2.224604 & 2.720564 \\ \mathrm{C} & -3.061498 & -0.483248 & -0.654740 \\ \mathrm{O} & -3.901661 & 0.255873 & -0.876686\end{array}$

$$
1 q=1 s:
$$

$\begin{array}{lrrr}\mathrm{P} & -0.371131 & 0.179795 & 0.054121 \\ \mathrm{C} & 0.189443 & 0.014475 & 1.776902 \\ \mathrm{H} & 1.241684 & -0.271407 & 1.817819 \\ \mathrm{H} & 0.063872 & 0.980868 & 2.265740 \\ \mathrm{H} & -0.407509 & -0.733515 & 2.298856 \\ \mathrm{O} & -0.269141 & -1.430496 & -0.338801 \\ \mathrm{~N} & -0.824257 & -1.709749 & -1.618899 \\ \mathrm{~W} & 0.704035 & 1.885665 & -1.428887 \\ \mathrm{C} & 1.786302 & 3.270546 & -2.456215 \\ \mathrm{O} & 2.424695 & 4.041206 & -3.015342 \\ \mathrm{C} & 0.782394 & 3.096174 & 0.241434 \\ \mathrm{O} & 0.829947 & 3.772166 & 1.163136 \\ \mathrm{C} & 2.467710 & 0.972086 & -0.875038 \\ \mathrm{O} & 3.437217 & 0.455339 & -0.556950\end{array}$

$\mathrm{E}=-2156.205162208 \mathrm{au}(\mathrm{COSMO}(\mathrm{tol}) / \mathrm{NCEPA} 1 /$ def2-TZVPP(ecp)) $\mathrm{ZPE}=0.16775073 \mathrm{au}$ 


$\begin{array}{lrllllll}\mathrm{C} & 1.482138 & -5.276992 & -1.152950 & \mathrm{O} & 2.597936 & -2.261518 & 0.837531 \\ \mathrm{O} & 1.022214 & -6.189376 & -0.645981 & \mathrm{C} & 3.195682 & -1.948101 & -2.949729 \\ \mathrm{C} & 2.472070 & -2.717220 & -0.200601 & \mathrm{O} & 3.673313 & -1.035529 & -3.439187\end{array}$

1r:

$\begin{array}{lrrr}\mathrm{P} & 0.081828 & -0.037693 & 0.068417 \\ \mathrm{C} & 1.623479 & 0.385790 & -0.822275 \\ \mathrm{H} & 2.456371 & -0.188995 & -0.414676 \\ \mathrm{H} & 1.489194 & 0.123112 & -1.871553 \\ \mathrm{H} & 1.832545 & 1.450828 & -0.729528 \\ \mathrm{~N} & 0.058998 & 0.903093 & 1.366690 \\ \mathrm{O} & 0.680193 & 1.803547 & 1.899344 \\ \mathrm{~W} & -1.515110 & -1.867922 & -0.396454 \\ \mathrm{C} & -2.776307 & -3.408097 & -0.813587\end{array}$

$\mathrm{E}=-1142.928734819$ au (COSMO(tol)/NCEPA1/def2-TZVPP(еср))

$\mathrm{ZPE}=0.08589586 \mathrm{au}$

$\begin{array}{lrrr}\mathrm{O} & -3.482273 & -4.278765 & -1.050028 \\ \mathrm{C} & -0.480366 & -2.255826 & -2.134758 \\ \mathrm{O} & 0.099930 & -2.463111 & -3.099128 \\ \mathrm{C} & -0.296247 & -3.184282 & 0.640578 \\ \mathrm{O} & 0.367135 & -3.919470 & 1.209442 \\ \mathrm{C} & -2.537988 & -1.435599 & 1.356718 \\ \mathrm{O} & -3.101323 & -1.191484 & 2.318313 \\ \mathrm{C} & -2.771440 & -0.572989 & -1.418941 \\ \mathrm{O} & -3.474186 & 0.128033 & -1.983151\end{array}$

$\begin{array}{lrrr} & \text { TS(1n } \rightarrow \text { 2): } & & \text { E }= \\ & & & \mathrm{ZPE} \\ & & & \\ & & & \\ \mathrm{P} & 0.062032 & -0.066792 & 0.120365 \\ \mathrm{C} & 1.551789 & 0.342874 & -0.800569 \\ \mathrm{H} & 2.061480 & -0.594084 & -1.036152 \\ \mathrm{H} & 1.288826 & 0.850763 & -1.729254 \\ \mathrm{H} & 2.166374 & 1.014486 & -0.202796 \\ \mathrm{O} & -0.216878 & 1.007047 & 1.223198 \\ \mathrm{O} & 0.115343 & 2.261838 & 0.522605 \\ \mathrm{~W} & -1.397377 & -1.892412 & -0.268249 \\ \mathrm{C} & -2.701104 & -3.454599 & -0.712829\end{array}$

$\mathrm{E}=-1163.463763504 \mathrm{au}$

$\mathrm{ZPE}=0.08437770 \mathrm{au}$

$v=-307.40 \mathrm{~cm}^{-1}$

$\begin{array}{lrrr}\text { 2: } & & & \mathrm{E}= \\ & & & \mathrm{ZP} \\ \mathrm{P} & 0.067638 & 0.050260 & 0.129324 \\ \mathrm{C} & 1.454805 & 0.453790 & -0.950898 \\ \mathrm{H} & 2.125290 & -0.406883 & -0.998867 \\ \mathrm{H} & 1.074397 & 0.642315 & -1.956987 \\ \mathrm{H} & 1.985241 & 1.327436 & -0.576167 \\ \mathrm{O} & -0.405406 & 1.480606 & 0.781211 \\ \mathrm{O} & 0.513950 & 0.567797 & 1.621837 \\ \mathrm{~W} & -1.403167 & -1.832665 & -0.282179 \\ \mathrm{C} & -2.650780 & -3.436470 & -0.621670\end{array}$

$\begin{array}{lrrr}\mathrm{O} & -3.410702 & -4.306843 & -0.967939 \\ \mathrm{C} & -0.459539 & -2.176274 & -2.083790 \\ \mathrm{O} & 0.075759 & -2.323677 & -3.081387 \\ \mathrm{C} & -0.082437 & -3.261100 & 0.592623 \\ \mathrm{O} & 0.635077 & -4.012592 & 1.057789 \\ \mathrm{C} & -2.296372 & -1.557829 & 1.580553 \\ \mathrm{O} & -2.786961 & -1.370362 & 2.589861 \\ \mathrm{C} & -2.790865 & -0.586891 & -1.112155 \\ \mathrm{O} & -3.560013 & 0.116616 & -1.566449\end{array}$

$\mathrm{E}=-1163.426782843 \mathrm{au}$

$\mathrm{ZPE}=0.08587820 \mathrm{au}$

$\mathrm{TS}(2 \rightarrow 3):$

$$
\begin{aligned}
& E=-1163.513661887 \mathrm{au} \\
& \mathrm{ZPE}=0.08413437 \mathrm{au} \\
& v=-405.70 \mathrm{~cm}^{-1}
\end{aligned}
$$

$\begin{array}{lrrr}\mathrm{O} & -3.341991 & -4.326005 & -0.810146 \\ \mathrm{C} & -0.427243 & -2.189608 & -2.060421 \\ \mathrm{O} & 0.128492 & -2.374654 & -3.043415 \\ \mathrm{C} & -0.076968 & -3.091879 & 0.693639 \\ \mathrm{O} & 0.655891 & -3.788519 & 1.223956 \\ \mathrm{C} & -2.343036 & -1.409963 & 1.520368 \\ \mathrm{O} & -2.856180 & -1.166524 & 2.509014 \\ \mathrm{C} & -2.751758 & -0.594155 & -1.257890 \\ \mathrm{O} & -3.494742 & 0.085288 & -1.795288\end{array}$

$\begin{array}{lll}0.133480 & 0.044004 & 0.231157\end{array}$

C 1.275433

0.631053

$-1.044131$ 


$\begin{array}{lrrrrrrr}\mathrm{H} & 1.961985 & -0.172447 & -1.316768 & \mathrm{C} & -0.264078 & -2.262682 & -1.967238 \\ \mathrm{H} & 0.711635 & 0.920181 & -1.933187 & \mathrm{O} & 0.353427 & -2.453292 & -2.909303 \\ \mathrm{H} & 1.833824 & 1.486315 & -0.663778 & \mathrm{C} & -0.142434 & -3.219665 & 0.813756 \\ \mathrm{O} & -0.487446 & 1.315430 & 0.935915 & \mathrm{O} & 0.493961 & -3.973122 & 1.378978 \\ \mathrm{O} & 0.873671 & 0.057050 & 1.621657 & \mathrm{C} & -2.291814 & -1.345357 & 1.515296 \\ \mathrm{~W} & -1.337082 & -1.866324 & -0.254768 & \mathrm{O} & -2.799167 & -1.051788 & 2.490500 \\ \mathrm{C} & -2.743264 & -3.370826 & -0.584784 & \mathrm{C} & -2.561201 & -0.615276 & -1.393079 \\ \mathrm{O} & -3.528850 & -4.178014 & -0.757331 & \mathrm{O} & -3.227649 & 0.044930 & -2.037468\end{array}$

$\begin{array}{lrrr}\text { 3: } & & \mathrm{E}= \\ & & & \mathrm{ZP} \\ \mathrm{P} & 0.094463 & 0.369533 & 0.235769 \\ \mathrm{C} & 0.922145 & 0.940075 & -1.258457 \\ \mathrm{H} & 1.320034 & 0.102309 & -1.824605 \\ \mathrm{H} & 0.218660 & 1.515822 & -1.857017 \\ \mathrm{H} & 1.735497 & 1.594771 & -0.938830 \\ \mathrm{O} & -0.555491 & 1.436450 & 1.011252 \\ \mathrm{O} & 0.764129 & -0.864335 & 0.822360 \\ \text { W } & -1.064452 & -1.948847 & -0.023095 \\ \mathrm{C} & -2.612098 & -3.117607 & -0.602526\end{array}$

$E=-1163.661571503 a u$

$\mathrm{ZPE}=0.08757674 \mathrm{au}$

$\operatorname{TS}(\mathbf{1 r} \rightarrow \mathbf{4}):$

$\mathrm{E}=-1142.859729506 \mathrm{au}(\mathrm{COSMO}(\mathrm{tol}) / \mathrm{NCEPA} 1 / \mathrm{def} 2-\mathrm{TZVPP}($ ecp$))$

$\mathrm{ZPE}=0.08370440 \mathrm{au}$

$v=-210.25 \mathrm{~cm}^{-1}$

$\begin{array}{lrrr}\mathrm{P} & -0.037104 & 0.142428 & -0.049079 \\ \mathrm{C} & 1.589497 & 0.298664 & -0.850787 \\ \mathrm{H} & 2.233382 & -0.534255 & -0.559648 \\ \mathrm{H} & 1.432611 & 0.272694 & -1.929869 \\ \mathrm{H} & 2.063907 & 1.240755 & -0.578944 \\ \mathrm{~N} & 0.520692 & 1.601572 & 1.861132 \\ \mathrm{O} & 0.424836 & 0.307788 & 1.609183 \\ \mathrm{~W} & -1.571680 & -1.731341 & -0.427412 \\ \mathrm{C} & -2.622123 & -3.468260 & -0.714455\end{array}$

$\begin{array}{lrrr}\mathrm{O} & -3.174863 & -4.457081 & -0.869897 \\ \mathrm{C} & -0.482679 & -2.131365 & -2.129916 \\ \mathrm{O} & 0.131935 & -2.335807 & -3.072197 \\ \mathrm{C} & -0.249385 & -2.822529 & 0.739535 \\ \mathrm{O} & 0.479961 & -3.421225 & 1.380383 \\ \mathrm{C} & -2.643802 & -1.253880 & 1.288129 \\ \mathrm{O} & -3.229034 & -0.985457 & 2.228897 \\ \mathrm{C} & -2.929822 & -0.654392 & -1.582649 \\ \mathrm{O} & -3.681896 & -0.078138 & -2.216984\end{array}$

4:

$\mathrm{E}=-1142.935710368 \mathrm{au}(\mathrm{COSMO}(\mathrm{tol}) / \mathrm{NCEPA} 1 /$ def2-TZVPP(ecp)) $\mathrm{ZPE}=0.08587898 \mathrm{au}$

$\begin{array}{lrrr}\mathrm{P} & 0.012036 & 0.122603 & 0.000283 \\ \mathrm{C} & 1.746682 & 0.132278 & -0.513979 \\ \mathrm{H} & 2.255708 & -0.719147 & -0.058132 \\ \mathrm{H} & 1.784185 & 0.012220 & -1.598733 \\ \mathrm{H} & 2.233841 & 1.061602 & -0.224397 \\ \mathrm{~N} & -0.432348 & 1.772596 & 0.220490 \\ \mathrm{O} & -0.028292 & 1.086473 & 1.383799 \\ \mathrm{~W} & -1.478291 & -1.772668 & -0.386010 \\ \mathrm{C} & -2.700417 & -3.384555 & -0.727287\end{array}$

$\begin{array}{lrrr}\mathrm{O} & -3.378356 & -4.285078 & -0.918112 \\ \mathrm{C} & -0.577643 & -2.058397 & -2.219219 \\ \mathrm{O} & -0.065434 & -2.210589 & -3.230719 \\ \mathrm{C} & -0.076153 & -3.018624 & 0.478983 \\ \mathrm{O} & 0.706608 & -3.705634 & 0.950855 \\ \mathrm{C} & -2.366350 & -1.452252 & 1.463961 \\ \mathrm{O} & -2.851973 & -1.269555 & 2.479428 \\ \mathrm{C} & -2.879817 & -0.508276 & -1.250527 \\ \mathrm{O} & -3.649555 & 0.187171 & -1.725259\end{array}$




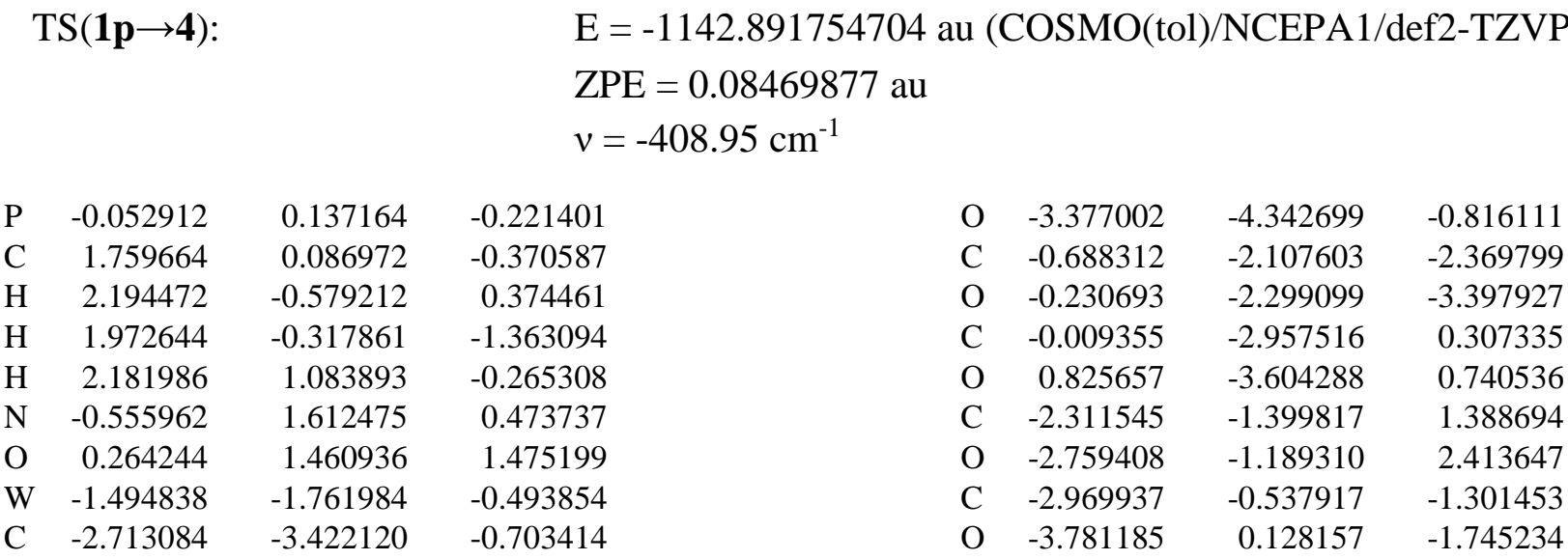

$\begin{array}{lrrr}\text { 5: } & & \mathrm{E} \\ & & & \mathrm{ZP} \\ & & & \\ \mathrm{P} & 0.000140 & 0.099850 & -0.277825 \\ \mathrm{C} & 0.322905 & -0.524199 & 1.359389 \\ \mathrm{H} & 1.057056 & -1.350400 & 1.285379 \\ \mathrm{H} & 0.803212 & 0.275867 & 1.955928 \\ \mathrm{H} & -0.555399 & -0.881626 & 1.893211 \\ \mathrm{O} & 1.022238 & 2.150067 & 0.317480 \\ \mathrm{~N} & 1.437534 & 2.244582 & 1.378769 \\ \mathrm{~N} & 2.124715 & -2.108477 & -0.411412 \\ \mathrm{O} & 1.596045 & -1.413781 & -1.150745 \\ \mathrm{~W} & -2.219538 & 0.161396 & -1.397619\end{array}$

$E=-1272.693521014 \mathrm{au}$

$\mathrm{ZPE}=0.090901770 \mathrm{au}$

$\mathrm{TS}(\mathbf{5} \rightarrow \mathbf{6}):$

$E=-1272.688194603 \mathrm{au}$

$\mathrm{ZPE}=0.09055592 \mathrm{au}$

$v=-70.45 \mathrm{~cm}^{-1}$

$\begin{array}{lrrr}\mathrm{P} & -0.038465 & -0.062309 & -0.392336 \\ \mathrm{C} & 0.415328 & -1.157924 & 0.949908 \\ \mathrm{H} & 1.355262 & -1.681625 & 0.724330 \\ \mathrm{H} & 0.629698 & -0.566064 & 1.857618 \\ \mathrm{H} & -0.371953 & -1.881224 & 1.152819 \\ \mathrm{O} & 0.459551 & 1.742215 & 0.940643 \\ \mathrm{~N} & 1.121752 & 1.563774 & 1.869246 \\ \mathrm{~N} & 2.918477 & -0.174621 & -0.000809 \\ \mathrm{O} & 2.224039 & 0.255711 & -0.801942 \\ \mathrm{~W} & -2.280437 & 0.175040 & -1.417278\end{array}$

$\begin{array}{lrrr}\mathrm{C} & -4.189246 & 0.279428 & -2.114774 \\ \mathrm{O} & -5.273032 & 0.323048 & -2.487100 \\ \mathrm{C} & -3.007491 & 0.126892 & 0.516282 \\ \mathrm{O} & -3.394353 & 0.096018 & 1.591578 \\ \mathrm{C} & -2.177596 & 2.245825 & -1.345122 \\ \mathrm{O} & -2.137708 & 3.387279 & -1.318374 \\ \mathrm{C} & -1.518001 & 0.232753 & -3.346808 \\ \mathrm{O} & -1.107044 & 0.267360 & -4.412143 \\ \mathrm{C} & -2.371875 & -1.887918 & -1.503641 \\ \mathrm{O} & -2.417290 & -3.029343 & -1.543873\end{array}$

6:

$E=-1272.890658558 \mathrm{au}$

$\mathrm{ZPE}=0.09622567 \mathrm{au}$

$\begin{array}{rrrr}\mathrm{P} & -0.032511 & -0.110501 & -0.116374 \\ \mathrm{C} & 0.119214 & 0.380064 & 1.618116 \\ \mathrm{H} & 1.126864 & 0.205137 & 1.995389 \\ \mathrm{H} & -0.127814 & 1.439466 & 1.703804\end{array}$

$\begin{array}{rrrr}\mathrm{H} & -0.600186 & -0.193970 & 2.204398 \\ \mathrm{O} & 1.334346 & 0.574264 & -0.806812 \\ \mathrm{~N} & 2.429891 & -0.304469 & -0.674398 \\ \mathrm{~N} & 2.117923 & -1.431400 & -0.328142\end{array}$

$\begin{array}{lrrr}\mathrm{C} & -4.079293 & 0.156909 & -2.227676 \\ \mathrm{O} & -5.135452 & 0.142857 & -2.675199 \\ \mathrm{C} & -3.004613 & -0.486091 & 0.395128 \\ \mathrm{O} & -3.407613 & -0.849271 & 1.402374 \\ \mathrm{C} & -2.485205 & 2.131899 & -0.813483 \\ \mathrm{O} & -2.637867 & 3.217877 & -0.490904 \\ \mathrm{C} & -1.356391 & 0.810285 & -3.165656 \\ \mathrm{O} & -0.882898 & 1.168888 & -4.142717 \\ \mathrm{C} & -1.953565 & -1.803548 & -1.993977 \\ \mathrm{O} & -1.806395 & -2.888770 & -2.322222\end{array}$




$\begin{array}{lrrr}\mathrm{O} & 0.731071 & -1.604664 & -0.136982 \\ \mathrm{~W} & -2.165140 & 0.084267 & -1.286228 \\ \mathrm{C} & -3.978664 & 0.235030 & -2.237471 \\ \mathrm{O} & -4.989638 & 0.317829 & -2.763899 \\ \mathrm{C} & -2.989695 & -1.226570 & 0.081531 \\ \mathrm{O} & -3.442316 & -1.946464 & 0.845487\end{array}$

$\begin{array}{lrrr}\mathrm{C} & -2.665474 & 1.670354 & -0.067101 \\ \mathrm{O} & -2.932823 & 2.546223 & 0.618444 \\ \mathrm{C} & -1.321307 & 1.394998 & -2.657498 \\ \mathrm{O} & -0.852724 & 2.112371 & -3.410417 \\ \mathrm{C} & -1.617390 & -1.507778 & -2.499141 \\ \mathrm{O} & -1.304012 & -2.379873 & -3.164482\end{array}$

$\mathrm{TS}\left(6 \rightarrow \mathrm{MePW}(\mathrm{CO})_{5}+\right.$ cyc-(NO) 2$): \quad \mathrm{E}=-1272.641060723 \mathrm{au}$

$\mathrm{ZPE}=0.09089971 \mathrm{au}$ $v=-511.80 \mathrm{~cm}^{-1}$

$\begin{array}{lrrr}\mathrm{P} & -0.053296 & 0.952138 & -0.397447 \\ \mathrm{C} & 0.090307 & 0.780024 & 1.420085 \\ \mathrm{H} & 1.137102 & 0.695621 & 1.713345 \\ \mathrm{H} & -0.296638 & 1.714107 & 1.845425 \\ \mathrm{H} & -0.482835 & -0.054420 & 1.824235 \\ \mathrm{O} & 2.009413 & -0.874805 & -0.875577 \\ \mathrm{~N} & 2.606308 & -1.717566 & 0.011716 \\ \mathrm{~N} & 1.651318 & -2.359472 & 0.428839 \\ \mathrm{O} & 0.623244 & -1.792289 & -0.254288 \\ \mathrm{~W} & -2.161563 & 0.360654 & -1.367478\end{array}$

$\begin{array}{lrrr}\mathrm{C} & -4.082772 & -0.030635 & -2.100039 \\ \mathrm{O} & -5.143198 & -0.249066 & -2.455063 \\ \mathrm{C} & -2.360818 & -1.121434 & 0.069897 \\ \mathrm{O} & -2.455789 & -1.912985 & 0.884150 \\ \mathrm{C} & -3.012600 & 1.693054 & -0.027543 \\ \mathrm{O} & -3.460407 & 2.416971 & 0.731986 \\ \mathrm{C} & -1.884235 & 1.843328 & -2.792773 \\ \mathrm{O} & -1.724384 & 2.652540 & -3.580687 \\ \mathrm{C} & -1.311542 & -1.005112 & -2.711780 \\ \mathrm{O} & -0.848000 & -1.736339 & -3.448778\end{array}$

$\mathrm{TS}\left(6 \rightarrow 7+\mathrm{N}_{2} \mathrm{O}\right)$
7:

$\begin{array}{lrrr}\mathrm{P} & -0.029499 & 0.715007 & -0.175834 \\ \mathrm{C} & 0.093950 & 0.650952 & 1.636194 \\ \mathrm{H} & 0.501789 & -0.327338 & 1.908337 \\ \mathrm{H} & 0.780931 & 1.421837 & 1.986242 \\ \mathrm{H} & -0.886956 & 0.764406 & 2.096424 \\ \mathrm{O} & 1.299781 & 1.027778 & -0.755224 \\ \mathrm{~W} & -2.120120 & 0.270614 & -1.290128 \\ \mathrm{C} & -3.918324 & -0.130587 & -2.230918 \\ \mathrm{O} & -4.910450 & -0.354783 & -2.747941\end{array}$

$$
\begin{aligned}
& E=-1272.868739531 \mathrm{au} \\
& \mathrm{ZPE}=0.09442348 \mathrm{au} \\
& v=-327.22 \mathrm{~cm}^{-1}
\end{aligned}
$$

$\begin{array}{lrrr}\mathrm{P} & 0.023754 & 0.128550 & -0.175618 \\ \mathrm{C} & 0.106951 & 0.413994 & 1.612522 \\ \mathrm{H} & 1.113607 & 0.239847 & 1.993127 \\ \mathrm{H} & -0.168026 & 1.455351 & 1.793198 \\ \mathrm{H} & -0.608650 & -0.230821 & 2.123503 \\ \mathrm{O} & 1.319739 & 0.660906 & -0.818330 \\ \mathrm{~N} & 2.652991 & -0.757963 & -0.543396 \\ \mathrm{~N} & 1.940062 & -1.615848 & -0.257201 \\ \mathrm{O} & 0.668281 & -1.680999 & -0.092098 \\ \mathrm{~W} & -2.159645 & 0.139000 & -1.319403 \\ \mathrm{C} & -4.004683 & 0.184029 & -2.201851 \\ \mathrm{O} & -5.040536 & 0.208001 & -2.687563\end{array}$

$\begin{array}{lrrr}\mathrm{C} & -2.849325 & -1.207601 & 0.080760 \\ \mathrm{O} & -3.221908 & -1.950760 & 0.867155 \\ \mathrm{C} & -2.721496 & 1.704797 & -0.099625 \\ \mathrm{O} & -3.026304 & 2.570821 & 0.583101 \\ \mathrm{C} & -1.412076 & 1.484007 & -2.712994 \\ \mathrm{O} & -0.994655 & 2.221505 & -3.476688 \\ \mathrm{C} & -1.559902 & -1.426241 & -2.540828 \\ \mathrm{O} & -1.218563 & -2.286260 & -3.209550 \\ \mathrm{C} & -1.884235 & 1.843328 & -2.792773 \\ \mathrm{O} & -1.724384 & 2.652540 & -3.580687 \\ \mathrm{C} & -1.311542 & -1.005112 & -2.711780 \\ \mathrm{O} & -0.848000 & -1.736339 & -3.448778\end{array}$

$\mathrm{E}=-1088.498197414 \mathrm{au}$

$\mathrm{ZPE}=0.08234484 \mathrm{au}$

$\begin{array}{lrrr}\mathrm{C} & -2.561709 & -1.127293 & 0.163459 \\ \mathrm{O} & -2.797564 & -1.892087 & 0.979640 \\ \mathrm{C} & -3.046419 & 1.717729 & -0.136301 \\ \mathrm{O} & -3.552928 & 2.511056 & 0.510776 \\ \mathrm{C} & -1.629292 & 1.685379 & -2.733285 \\ \mathrm{O} & -1.349698 & 2.460684 & -3.520055 \\ \mathrm{C} & -1.177495 & -1.171894 & -2.454428 \\ \mathrm{O} & -0.653398 & -1.957942 & -3.091733\end{array}$




\begin{tabular}{rrrrrrrr}
\multicolumn{7}{c}{$\left.\mathrm{MePW}^{2} \mathrm{CO}\right)_{5} \cdot \mathrm{O}=\mathrm{CMe}_{2}:$} & \multicolumn{2}{c}{$\mathrm{E}=-1206.378694243 \mathrm{au}$ (optimized with CPCM(tol)/PBEh-3c } \\
\multicolumn{7}{c}{$\mathrm{ZPE}=0.16795137 \mathrm{au}$} \\
$\mathrm{P}$ & 0.863703 & -0.553247 & 1.549293 & $\mathrm{O}$ & -1.789337 & 0.410344 & -1.299462 \\
$\mathrm{C}$ & 2.053088 & -0.489787 & 0.146900 & $\mathrm{C}$ & -1.971749 & 0.107197 & 2.532818 \\
$\mathrm{H}$ & 2.972367 & -0.052165 & 0.555923 & $\mathrm{O}$ & -2.703393 & -0.564459 & 3.091092 \\
$\mathrm{H}$ & 2.317608 & -1.495290 & -0.181918 & $\mathrm{O}$ & 0.821504 & 1.744619 & -2.518908 \\
$\mathrm{H}$ & 1.746894 & 0.118318 & -0.702181 & $\mathrm{C}$ & 0.305279 & 1.239227 & -3.488637 \\
$\mathrm{~W}$ & -0.626084 & 1.316676 & 1.551281 & $\mathrm{C}$ & 0.444505 & -0.225426 & -3.803716 \\
$\mathrm{C}$ & -2.006045 & 2.880936 & 1.566564 & $\mathrm{H}$ & 0.906803 & -0.770309 & -2.983717 \\
$\mathrm{O}$ & -2.756740 & 3.736201 & 1.556351 & $\mathrm{H}$ & -0.525070 & -0.668738 & -4.034199 \\
$\mathrm{C}$ & 0.054028 & 1.926310 & 3.416533 & $\mathrm{H}$ & 1.062554 & -0.345960 & -4.696454 \\
$\mathrm{O}$ & 0.416729 & 2.251168 & 4.443847 & $\mathrm{C}$ & -0.525701 & 2.037775 & -4.456477 \\
$\mathrm{C}$ & 0.825189 & 2.476479 & 0.632166 & $\mathrm{H}$ & -0.249037 & 1.816937 & -5.488168 \\
$\mathrm{O}$ & 1.652342 & 3.109354 & 0.177482 & $\mathrm{H}$ & -1.574896 & 1.753243 & -4.346619 \\
$\mathrm{C}$ & -1.361548 & 0.743723 & -0.299091 & $\mathrm{H}$ & -0.433089 & 3.105855 & -4.273722
\end{tabular}

$\mathrm{TS}\left(\mathrm{MePW}(\mathrm{CO})_{5}+\mathrm{O}=\mathrm{CMe}_{2} \rightarrow \mathbf{1 f}\right): \mathrm{E}=-1206.379292182$ au (optimized with CPCM(tol)/PBEh-3c(ecp)) $\mathrm{ZPE}=0.16805482 \mathrm{au}$ $v=-22.82 \mathrm{~cm}^{-1}$

$\begin{array}{lrrr}\mathrm{P} & 0.750594 & -0.611782 & 1.259722 \\ \mathrm{C} & 2.190620 & -0.312758 & 0.153377 \\ \mathrm{H} & 2.936556 & 0.237517 & 0.738066 \\ \mathrm{H} & 2.651887 & -1.247280 & -0.163053 \\ \mathrm{H} & 1.949818 & 0.298692 & -0.715845 \\ \mathrm{~W} & -0.659284 & 1.307441 & 1.492239 \\ \mathrm{C} & -2.005034 & 2.878851 & 1.747282 \\ \mathrm{O} & -2.739697 & 3.738242 & 1.879065 \\ \mathrm{C} & 0.126801 & 1.755672 & 3.366373 \\ \mathrm{O} & 0.552460 & 2.000983 & 4.390735 \\ \mathrm{C} & 0.778638 & 2.516187 & 0.629441 \\ \mathrm{O} & 1.590949 & 3.184861 & 0.197865 \\ \mathrm{C} & -1.517918 & 0.985552 & -0.369927\end{array}$

$\begin{array}{lrrr}\mathrm{O} & -2.014453 & 0.806158 & -1.377320 \\ \mathrm{C} & -1.981705 & 0.008538 & 2.382968 \\ \mathrm{O} & -2.700621 & -0.720846 & 2.882255 \\ \mathrm{O} & 0.854281 & 1.720307 & -2.519775 \\ \mathrm{C} & 0.311239 & 1.168544 & -3.448899 \\ \mathrm{C} & 0.308469 & -0.328004 & -3.605474 \\ \mathrm{H} & 0.671910 & -0.825932 & -2.709153 \\ \mathrm{H} & -0.689664 & -0.695172 & -3.846939 \\ \mathrm{H} & 0.953623 & -0.603639 & -4.443049 \\ \mathrm{C} & -0.409701 & 1.938067 & -4.522251 \\ \mathrm{H} & -0.132685 & 1.580790 & -5.514921 \\ \mathrm{H} & -1.484979 & 1.773570 & -4.419919 \\ \mathrm{H} & -0.211323 & 3.004823 & -4.448824\end{array}$

\section{1f $\cdot \mathrm{O}=\mathrm{CMe}_{2}$ :}

$\begin{array}{lrrr}\mathrm{P} & 0.000140 & 0.099850 & -0.277825 \\ \mathrm{C} & 0.322905 & -0.524199 & 1.359389 \\ \mathrm{H} & 1.057056 & -1.350400 & 1.285379 \\ \mathrm{H} & 0.803212 & 0.275867 & 1.955928 \\ \mathrm{H} & -0.555399 & -0.881626 & 1.893211 \\ \mathrm{O} & 1.022238 & 2.150067 & 0.317480 \\ \mathrm{~N} & 1.437534 & 2.244582 & 1.378769 \\ \mathrm{~N} & 2.124715 & -2.108477 & -0.411412 \\ \mathrm{O} & 1.596045 & -1.413781 & -1.150745 \\ \mathrm{~W} & -2.219538 & 0.161396 & -1.397619\end{array}$

$\mathrm{E}=-1399.008065930 \mathrm{au}$

$\mathrm{ZPE}=0.24905996 \mathrm{au}$ 
$\mathrm{TS}\left(\mathbf{1 f} \cdot \mathrm{O}=\mathrm{CMe}_{2} \rightarrow \mathbf{1 f} \cdot \mathrm{O}=\mathrm{CMe}_{2}\right): \quad \mathrm{E}=-1399.002652116 \mathrm{au}$

$$
\begin{aligned}
& \mathrm{ZPE}=0.24948750 \mathrm{au} \\
& v=-134.95 \mathrm{~cm}^{-1}
\end{aligned}
$$

$\begin{array}{lrrr}\mathrm{P} & -0.397154 & 0.664200 & 0.914829 \\ \mathrm{C} & 1.457612 & 0.614994 & 0.857137 \\ \mathrm{H} & 1.884979 & 1.156562 & 1.702074 \\ \mathrm{H} & 1.796168 & -0.418147 & 0.888881 \\ \mathrm{H} & 1.806434 & 1.067365 & -0.068466 \\ \mathrm{~W} & -1.343924 & -0.665806 & -1.181299 \\ \mathrm{C} & -2.084289 & -1.666785 & -2.765414 \\ \mathrm{O} & -2.501797 & -2.240683 & -3.674703 \\ \mathrm{C} & 0.606127 & -1.062091 & -1.733504 \\ \mathrm{O} & 1.694220 & -1.272867 & -2.023452 \\ \mathrm{C} & -1.396849 & -2.404142 & -0.090581 \\ \mathrm{O} & -1.455561 & -3.378245 & 0.517546 \\ \mathrm{C} & -3.210869 & -0.188947 & -0.487294 \\ \mathrm{O} & -4.245886 & 0.092608 & -0.069560 \\ \mathrm{C} & -1.326767 & 1.049193 & -2.311194 \\ \mathrm{O} & -1.346375 & 2.006230 & -2.947916 \\ \mathrm{O} & -0.174631 & -1.081705 & 2.236960 \\ \mathrm{C} & -1.040513 & -1.537842 & 2.980563\end{array}$

$\operatorname{TS}(\mathbf{1 f} \rightarrow \mathbf{8}):$

$$
\begin{aligned}
& E=-1206.135142146 \mathrm{au} \\
& \mathrm{ZPE}=0.16258738 \mathrm{au} \\
& v=-178.54 \mathrm{~cm}^{-1}
\end{aligned}
$$

$\begin{array}{lrrr}\mathrm{C} & -2.434456 & -0.999923 & 2.989408 \\ \mathrm{H} & -2.943845 & -1.216708 & 3.926584 \\ \mathrm{H} & -2.422385 & 0.070246 & 2.784055 \\ \mathrm{H} & -2.990957 & -1.484411 & 2.182287 \\ \mathrm{C} & -0.708641 & -2.678319 & 3.887687 \\ \mathrm{H} & -0.818531 & -2.355014 & 4.926926 \\ \mathrm{H} & -1.424592 & -3.488693 & 3.729952 \\ \mathrm{H} & 0.304160 & -3.032140 & 3.712256 \\ \mathrm{O} & -0.147737 & 2.599827 & -0.095408 \\ \mathrm{C} & -1.005617 & 3.473726 & -0.201473 \\ \mathrm{C} & -2.412342 & 3.256482 & 0.252010 \\ \mathrm{H} & -2.915742 & 4.197519 & 0.466564 \\ \mathrm{H} & -2.956562 & 2.759629 & -0.556025 \\ \mathrm{H} & -2.428304 & 2.593538 & 1.116656 \\ \mathrm{C} & -0.649162 & 4.786698 & -0.820107 \\ \mathrm{H} & -0.760153 & 5.576774 & -0.071521 \\ \mathrm{H} & 0.369596 & 4.773917 & -1.199105 \\ \mathrm{H} & -1.350697 & 5.013086 & -1.626695\end{array}$

$\begin{array}{lrrr}\mathrm{P} & 0.184123 & -0.394223 & 1.031679 \\ \mathrm{C} & 1.781818 & -0.002072 & 0.208809 \\ \mathrm{H} & 1.768650 & 0.932177 & -0.349662 \\ \mathrm{H} & 2.500147 & 0.096761 & 1.030859 \\ \mathrm{H} & 2.120420 & -0.816859 & -0.430084 \\ \mathrm{~W} & -0.794932 & 1.610625 & 2.145641 \\ \mathrm{C} & -1.497692 & 3.233628 & 3.176640 \\ \mathrm{O} & -1.874906 & 4.152046 & 3.751855 \\ \mathrm{C} & -1.387161 & 0.344778 & 3.661607 \\ \mathrm{O} & -1.720239 & -0.376586 & 4.486852 \\ \mathrm{C} & 1.052387 & 1.884070 & 3.022142 \\ \mathrm{O} & 2.086604 & 2.032095 & 3.491516 \\ \mathrm{C} & -0.176696 & 2.740539 & 0.532086\end{array}$

$\mathrm{TS}(\mathbf{1 f} \rightarrow \mathbf{8})$ :

$\begin{array}{lrrr}\mathrm{O} & 0.181974 & 3.326988 & -0.382637 \\ \mathrm{C} & -2.628573 & 1.330157 & 1.222321 \\ \mathrm{O} & -3.634274 & 1.163207 & 0.703802 \\ \mathrm{O} & -0.829453 & -0.358156 & -0.925925 \\ \mathrm{C} & -1.034827 & -1.592257 & -0.882013 \\ \mathrm{C} & -2.293819 & -2.134100 & -0.283407 \\ \mathrm{H} & -2.689526 & -1.460447 & 0.471590 \\ \mathrm{H} & -3.029859 & -2.204933 & -1.093237 \\ \mathrm{H} & -2.150555 & -3.133259 & 0.124860 \\ \mathrm{C} & -0.125548 & -2.548807 & -1.585504 \\ \mathrm{H} & 0.172607 & -3.355056 & -0.913621 \\ \mathrm{H} & -0.683467 & -3.010009 & -2.407453 \\ \mathrm{H} & 0.746836 & -2.038969 & -1.984912\end{array}$

$\mathrm{E}=-1206.368712448$ au (optimized with CPCM(tol)/PBEh-3c(ecp)) $\mathrm{ZPE}=0.16935121 \mathrm{au}$ $v=-244.52 \mathrm{~cm}^{-1}$

$\begin{array}{lrrr}\mathrm{P} & 0.264596 & -0.399236 & 0.886285 \\ \mathrm{C} & 1.866556 & 0.071625 & 0.110603 \\ \mathrm{H} & 1.805927 & 0.995450 & -0.463757 \\ \mathrm{H} & 2.570440 & 0.229846 & 0.930878 \\ \mathrm{H} & 2.278794 & -0.715760 & -0.520400 \\ \mathrm{~W} & -0.773005 & 1.569433 & 2.110191\end{array}$

$\begin{array}{lrrr}\text { C } & -1.525111 & 3.135063 & 3.135382 \\ \text { O } & -1.944428 & 4.034570 & 3.710718 \\ \text { C } & -1.208892 & 0.291563 & 3.659895 \\ \text { O } & -1.454112 & -0.427446 & 4.512837 \\ \text { C } & 1.076769 & 1.955884 & 2.919309 \\ \text { O } & 2.103835 & 2.178253 & 3.367209\end{array}$




$\begin{array}{lrrrrrrr}\mathrm{C} & -0.313775 & 2.748903 & 0.489243 & \mathrm{H} & -2.630147 & -2.064926 & 0.496406 \\ \mathrm{O} & -0.054654 & 3.387013 & -0.421377 & \mathrm{H} & -3.133772 & -1.245338 & -0.970683 \\ \mathrm{C} & -2.623929 & 1.207845 & 1.290153 & \mathrm{H} & -2.691187 & -2.981750 & -1.007746 \\ \mathrm{O} & -3.654733 & 1.018726 & 0.834803 & \mathrm{C} & -0.078966 & -2.673634 & -1.187276 \\ \mathrm{O} & -0.742951 & -0.387071 & -0.731126 & \mathrm{H} & -0.171802 & -3.550153 & -0.547423 \\ \mathrm{C} & -1.043401 & -1.619882 & -0.786444 & \mathrm{H} & -0.371011 & -2.993066 & -2.195134 \\ \mathrm{C} & -2.459106 & -2.007858 & -0.584070 & \mathrm{H} & 0.952099 & -2.336715 & -1.214670\end{array}$

$\begin{array}{lrrr}\text { 8: } & & \mathrm{E}= \\ & & & \mathrm{ZP} \\ & & & \\ \mathrm{P} & -0.056364 & -0.012906 & 0.172005 \\ \mathrm{C} & 1.710066 & 0.060801 & -0.254400 \\ \mathrm{H} & 1.953942 & 1.095200 & -0.501630 \\ \mathrm{H} & 2.291517 & -0.223733 & 0.624618 \\ \mathrm{H} & 1.969221 & -0.579431 & -1.093212 \\ \mathrm{~W} & -0.877797 & 1.572790 & 1.908058 \\ \mathrm{C} & -1.514936 & 2.917140 & 3.303259 \\ \mathrm{O} & -1.871484 & 3.677431 & 4.083789 \\ \mathrm{C} & -1.598757 & 0.009425 & 3.051980 \\ \mathrm{O} & -1.989712 & -0.859044 & 3.686022 \\ \mathrm{C} & 0.941972 & 1.410492 & 2.859289 \\ \mathrm{O} & 1.958315 & 1.313215 & 3.378712 \\ \mathrm{C} & -0.129403 & 3.110591 & 0.750987\end{array}$$$
\mathrm{ZPE}=0.165943880 \mathrm{au}
$$

8:

$\begin{array}{lrrr}\mathrm{P} & -0.054089 & -0.025340 & 0.190466 \\ \mathrm{C} & 1.707216 & 0.044047 & -0.261659 \\ \mathrm{H} & 1.972242 & 1.079920 & -0.473729 \\ \mathrm{H} & 2.303295 & -0.282701 & 0.591147 \\ \mathrm{H} & 1.958804 & -0.564002 & -1.126444 \\ \mathrm{~W} & -0.872729 & 1.567425 & 1.905731 \\ \mathrm{C} & -1.513758 & 2.914834 & 3.279588 \\ \mathrm{O} & -1.872955 & 3.678385 & 4.050568 \\ \mathrm{C} & -1.609526 & 0.030843 & 3.066510 \\ \mathrm{O} & -2.012012 & -0.813357 & 3.718480 \\ \mathrm{C} & 0.931191 & 1.424900 & 2.879354 \\ \mathrm{O} & 1.933707 & 1.349883 & 3.419416 \\ \mathrm{C} & -0.123790 & 3.107501 & 0.762588\end{array}$

$\operatorname{TS}(\mathbf{8} \rightarrow \mathbf{9}):$
$\begin{array}{lr}\mathrm{P} & -0.244826 \\ \mathrm{C} & 1.562147 \\ \mathrm{H} & 1.902065 \\ \mathrm{H} & 2.082427 \\ \mathrm{H} & 1.802922\end{array}$
0.206844
0.136444
1.162560
$-0.271401$
$-0.438457$
$-0.243076$
$-0.512687$
$-0.659140$
0.354750
$-1.407249$

$\mathrm{E}=-1206.431559684 \mathrm{au}$ (optimized with CPCM(tol)/PBEh-3c(ecp)) $\mathrm{ZPE}=0.17245271 \mathrm{au}$

$\begin{array}{lrrr}\mathrm{O} & 0.296046 & 3.964266 & 0.138190 \\ \mathrm{C} & -2.676961 & 1.711704 & 0.910171 \\ \mathrm{O} & -3.672614 & 1.794655 & 0.363850 \\ \mathrm{O} & -0.901691 & -0.307609 & -1.224876 \\ \mathrm{C} & -0.802132 & -1.524895 & -0.448553 \\ \mathrm{C} & -2.112107 & -1.982188 & 0.138472 \\ \mathrm{H} & -2.668868 & -1.172841 & 0.607708 \\ \mathrm{H} & -2.741158 & -2.407370 & -0.646623 \\ \mathrm{H} & -1.942035 & -2.754704 & 0.887837 \\ \mathrm{C} & -0.012289 & -2.629098 & -1.099137 \\ \mathrm{H} & 0.224084 & -3.402069 & -0.368466 \\ \mathrm{H} & -0.611723 & -3.089613 & -1.887760 \\ \mathrm{H} & 0.917888 & -2.291239 & -1.549026\end{array}$

$$
\begin{aligned}
& E=-1206.144734023 \mathrm{au} \\
& \mathrm{ZPE}=0.16325546 \mathrm{au} \\
& v=-172.03 \mathrm{~cm}^{-1}
\end{aligned}
$$

$\begin{array}{lrrr}\mathrm{O} & 0.297314 & 3.956576 & 0.109280 \\ \mathrm{C} & -2.689067 & 1.696227 & 0.912382 \\ \mathrm{O} & -3.685435 & 1.755045 & 0.356283 \\ \mathrm{O} & -0.881621 & -0.299660 & -1.242009 \\ \mathrm{C} & -0.792435 & -1.535907 & -0.425725 \\ \mathrm{C} & -2.124605 & -1.959743 & 0.136622 \\ \mathrm{H} & -2.658710 & -1.127385 & 0.589298 \\ \mathrm{H} & -2.745012 & -2.369280 & -0.664846 \\ \mathrm{H} & -1.983899 & -2.733789 & 0.892569 \\ \mathrm{C} & -0.008337 & -2.643130 & -1.077035 \\ \mathrm{H} & 0.216270 & -3.420510 & -0.344679 \\ \mathrm{H} & -0.610963 & -3.091157 & -1.871998 \\ \mathrm{H} & 0.923956 & -2.297920 & -1.515815\end{array}$




$\begin{array}{rrrr}\mathrm{C} & 0.931721 & 1.026847 & 2.684195 \\ \mathrm{O} & 1.951097 & 0.759428 & 3.136091 \\ \mathrm{C} & -0.044933 & 3.100061 & 0.884297 \\ \mathrm{O} & 0.439661 & 3.992332 & 0.359806 \\ \mathrm{C} & -2.704438 & 1.802825 & 0.901603 \\ \mathrm{O} & -3.706582 & 1.963426 & 0.376037 \\ \mathrm{O} & -0.939271 & 0.395016 & -1.598861 \\ \mathrm{C} & -0.749649 & -1.534991 & -0.169148\end{array}$

$\begin{array}{rrrr}\mathrm{C} & -2.173990 & -1.833435 & -0.002606 \\ \mathrm{H} & -2.804939 & -0.951428 & 0.039267 \\ \mathrm{H} & -2.513600 & -2.517321 & -0.786877 \\ \mathrm{H} & -2.272927 & -2.396797 & 0.938114 \\ \mathrm{C} & 0.165481 & -2.650995 & -0.435684 \\ \mathrm{H} & 1.079761 & -2.563774 & 0.155634 \\ \mathrm{H} & -0.294118 & -3.629901 & -0.305707 \\ \mathrm{H} & 0.486773 & -2.554752 & -1.482625\end{array}$

$\mathrm{TS}(\mathbf{8} \rightarrow \mathbf{9}):$

$\mathrm{E}=-1206.380747140 \mathrm{au}($ optimized with CPCM(tol)/PBEh-3c(ecp))

$\mathrm{ZPE}=0.16890221 \mathrm{au}$

$v=-172.03 \mathrm{~cm}^{-1}$

$\begin{array}{lrrr}\mathrm{P} & -0.257499 & 0.213825 & -0.247297 \\ \mathrm{C} & 1.546545 & 0.115031 & -0.545803 \\ \mathrm{H} & 1.907595 & 1.131723 & -0.704984 \\ \mathrm{H} & 2.087219 & -0.293903 & 0.307561 \\ \mathrm{H} & 1.777987 & -0.463582 & -1.440579 \\ \mathrm{~W} & -0.881530 & 1.459811 & 1.828736 \\ \mathrm{C} & -1.413166 & 2.648724 & 3.390650 \\ \mathrm{O} & -1.711753 & 3.328256 & 4.260748 \\ \mathrm{C} & -1.693941 & -0.155853 & 2.795252 \\ \mathrm{O} & -2.149410 & -1.049788 & 3.344566 \\ \mathrm{C} & 0.933443 & 1.039490 & 2.686157 \\ \mathrm{O} & 1.945178 & 0.793892 & 3.156873 \\ \mathrm{C} & -0.054802 & 3.116025 & 0.911664\end{array}$

$\begin{array}{lrrr}\mathrm{O} & 0.408298 & 4.034980 & 0.422269 \\ \mathrm{C} & -2.689308 & 1.820224 & 0.900769 \\ \mathrm{O} & -3.689457 & 2.007875 & 0.388307 \\ \mathrm{O} & -0.980311 & 0.420624 & -1.586757 \\ \mathrm{C} & -0.746846 & -1.543547 & -0.153513 \\ \mathrm{C} & -2.172143 & -1.847656 & -0.035927 \\ \mathrm{H} & -2.814480 & -0.977184 & 0.046497 \\ \mathrm{H} & -2.484988 & -2.463929 & -0.884886 \\ \mathrm{H} & -2.303247 & -2.491311 & 0.842183 \\ \mathrm{C} & 0.174157 & -2.655762 & -0.384272 \\ \mathrm{H} & 1.094108 & -2.544986 & 0.190967 \\ \mathrm{H} & -0.269379 & -3.637377 & -0.228804 \\ \mathrm{H} & 0.481770 & -2.584262 & -1.436575\end{array}$

9:

$\mathrm{E}=-1206.204158570 \mathrm{au}$

$\mathrm{ZPE}=0.16646746 \mathrm{au}$

$\begin{array}{lrrr}\mathrm{P} & -0.238470 & 0.330626 & -0.737076 \\ \mathrm{C} & 1.550856 & 0.366608 & -1.000772 \\ \mathrm{H} & 1.852155 & 1.404324 & -1.138300 \\ \mathrm{H} & 2.101281 & -0.066062 & -0.169957 \\ \mathrm{H} & 1.765225 & -0.186358 & -1.916628 \\ \mathrm{~W} & -0.892073 & 0.952708 & 1.704030 \\ \mathrm{C} & -1.240590 & 2.151894 & 3.319679 \\ \mathrm{O} & -1.434880 & 2.825496 & 4.222460 \\ \mathrm{C} & -1.751269 & -0.636222 & 2.733006 \\ \mathrm{O} & -2.211448 & -1.520756 & 3.285611 \\ \mathrm{C} & 0.960890 & 0.442912 & 2.431322 \\ \mathrm{O} & 1.999756 & 0.169839 & 2.827096 \\ \mathrm{C} & -0.053502 & 2.641803 & 0.860888\end{array}$

$\begin{array}{lrrr}\mathrm{O} & 0.421554 & 3.578930 & 0.413698 \\ \mathrm{C} & -2.739231 & 1.455239 & 0.896087 \\ \mathrm{O} & -3.752690 & 1.747069 & 0.464760 \\ \mathrm{O} & -1.022763 & 0.971589 & -1.828428 \\ \mathrm{C} & -0.802855 & -1.063343 & 0.103146 \\ \mathrm{C} & -2.178458 & -1.570580 & -0.273787 \\ \mathrm{H} & -2.816027 & -0.789436 & -0.681023 \\ \mathrm{H} & -2.065417 & -2.337313 & -1.048071 \\ \mathrm{H} & -2.686296 & -2.036450 & 0.572335 \\ \mathrm{C} & 0.142702 & -2.130490 & 0.607261 \\ \mathrm{H} & 1.129491 & -1.755361 & 0.865285 \\ \mathrm{H} & -0.262546 & -2.637254 & 1.485144 \\ \mathrm{H} & 0.268643 & -2.888075 & -0.173961\end{array}$

9:

$\mathrm{E}=-1206.444799473 \mathrm{au}$ (optimized with CPCM(tol)/PBEh-3c(ecp)) $\mathrm{ZPE}=0.17238171 \mathrm{au}$

$\begin{array}{rrrr}\mathrm{P} & -0.247146 & 0.340579 & -0.742142 \\ \mathrm{C} & 1.540133 & 0.366159 & -1.031492 \\ \mathrm{H} & 1.850090 & 1.391659 & -1.224330 \\ \mathrm{H} & 2.113548 & -0.024720 & -0.196120\end{array}$

$\begin{array}{lrrr}\mathrm{H} & 1.754603 & -0.227715 & -1.920590 \\ \mathrm{~W} & -0.884451 & 0.918033 & 1.676863 \\ \mathrm{C} & -1.254874 & 2.116161 & 3.283649 \\ \mathrm{O} & -1.465285 & 2.784961 & 4.181185\end{array}$




$\begin{array}{lrrr}\mathrm{C} & -1.778639 & -0.629287 & 2.740267 \\ \mathrm{O} & -2.258359 & -1.471803 & 3.331550 \\ \mathrm{C} & 0.944951 & 0.415280 & 2.456408 \\ \mathrm{O} & 1.964522 & 0.164751 & 2.901773 \\ \mathrm{C} & -0.032434 & 2.607350 & 0.875359 \\ \mathrm{O} & 0.445138 & 3.555701 & 0.463058 \\ \mathrm{C} & -2.715365 & 1.455769 & 0.867502 \\ \mathrm{O} & -3.721782 & 1.782577 & 0.454100 \\ \mathrm{O} & -1.056830 & 1.008092 & -1.808374\end{array}$

$\begin{array}{lrrr}\mathrm{C} & -0.803385 & -1.033742 & 0.134308 \\ \mathrm{C} & -2.165391 & -1.570614 & -0.252033 \\ \mathrm{H} & -2.841101 & -0.810491 & -0.636545 \\ \mathrm{H} & -2.039634 & -2.314554 & -1.044338 \\ \mathrm{H} & -2.660080 & -2.073564 & 0.579373 \\ \mathrm{C} & 0.156183 & -2.105631 & 0.600068 \\ \mathrm{H} & 1.156917 & -1.744554 & 0.824991 \\ \mathrm{H} & -0.213293 & -2.614692 & 1.491240 \\ \mathrm{H} & 0.256002 & -2.864369 & -0.181924\end{array}$

$\begin{array}{ll}\mathrm{TS}(\mathbf{1 g} \rightarrow \mathbf{1 0}): & \mathrm{E}=-1201.606696416 \mathrm{au} \\ & \mathrm{ZPE}=0.08956279 \mathrm{au} \\ & v=-264.02 \mathrm{~cm}^{-1}\end{array}$

$\begin{array}{lrrr}\mathrm{P} & -0.435346 & 0.093562 & 0.136195 \\ \mathrm{~W} & -0.153282 & 0.001608 & 2.518223 \\ \mathrm{C} & 0.096363 & -0.072416 & 4.586169 \\ \mathrm{O} & 0.240326 & -0.134520 & 5.715411 \\ \mathrm{C} & -1.512079 & -1.558943 & 2.597763 \\ \mathrm{O} & -2.248791 & -2.430952 & 2.609228 \\ \mathrm{C} & 1.333757 & -1.414549 & 2.239278 \\ \mathrm{O} & 2.133996 & -2.204249 & 2.047072 \\ \mathrm{C} & 1.221699 & 1.557987 & 2.379982 \\ \mathrm{O} & 1.966953 & 2.414180 & 2.283831\end{array}$

$\begin{array}{rrrr}\mathrm{C} & -1.643859 & 1.433315 & 2.741536 \\ \mathrm{O} & -2.453537 & 2.227262 & 2.861554 \\ \mathrm{C} & -0.484588 & -1.513854 & -0.741191 \\ \mathrm{H} & 0.186001 & -2.251349 & -0.301247 \\ \mathrm{H} & -0.276828 & -1.396834 & -1.803285 \\ \mathrm{H} & -1.510777 & -1.882322 & -0.630286 \\ \mathrm{O} & 1.588144 & 0.815724 & -1.061733 \\ \mathrm{C} & 0.601173 & 1.456583 & -1.325443 \\ \mathrm{O} & 0.033308 & 2.319549 & -1.887382\end{array}$

10:

$\mathrm{E}=-1201.632419197 \mathrm{au}$

$\mathrm{ZPE}=0.09185562 \mathrm{au}$

$\begin{array}{lrrr}\mathrm{P} & -0.027458 & -0.031279 & 0.071459 \\ \mathrm{~W} & 0.013033 & -0.011409 & 2.509310 \\ \mathrm{C} & 0.043609 & -0.015500 & 4.561845 \\ \mathrm{O} & 0.061928 & -0.024859 & 5.704300 \\ \mathrm{C} & -1.210310 & -1.671232 & 2.516545 \\ \mathrm{O} & -1.886793 & -2.593347 & 2.504037 \\ \mathrm{C} & 1.680547 & -1.244984 & 2.492993 \\ \mathrm{O} & 2.594243 & -1.928474 & 2.487492 \\ \mathrm{C} & 1.246417 & 1.655250 & 2.444071 \\ \mathrm{O} & 1.923502 & 2.572112 & 2.396264\end{array}$

$\begin{array}{rrrr}\mathrm{C} & -1.646885 & 1.230592 & 2.554831 \\ \mathrm{O} & -2.559844 & 1.914872 & 2.586678 \\ \mathrm{C} & -0.853720 & -1.324814 & -0.896421 \\ \mathrm{H} & -0.447375 & -2.293928 & -0.602057 \\ \mathrm{H} & -0.729114 & -1.172858 & -1.966599 \\ \mathrm{H} & -1.915240 & -1.305124 & -0.641345 \\ \mathrm{O} & 1.451700 & 0.297599 & -0.874983 \\ \mathrm{C} & 0.504856 & 1.216603 & -1.104874 \\ \mathrm{O} & 0.439534 & 2.190562 & -1.777871\end{array}$

$\mathrm{TS}\left(\mathbf{1} \mathbf{k} \rightarrow \mathbf{7}+\mathrm{NMe}_{3}\right)$ :

$$
\begin{aligned}
& E=-1262.581447274 \mathrm{au} \\
& \mathrm{ZPE}=0.20481725 \mathrm{au} \\
& v=-452.26 \mathrm{~cm}^{-1}
\end{aligned}
$$

$\begin{array}{lrrr}\mathrm{P} & -0.455392 & -0.042161 & -0.031524 \\ \mathrm{C} & 1.375892 & -0.210549 & -0.301815 \\ \mathrm{H} & 1.861225 & 0.761027 & -0.405215 \\ \mathrm{H} & 1.785414 & -0.708100 & 0.580526 \\ \mathrm{H} & 1.612851 & -0.828928 & -1.169135 \\ \mathrm{~W} & -0.864738 & 1.676338 & 1.904687 \\ \mathrm{C} & -1.179829 & 2.995718 & 3.393615 \\ \mathrm{O} & -1.355388 & 3.761635 & 4.238951\end{array}$

$\begin{array}{lrrr}\text { C } & 0.829033 & 0.949734 & 2.824973 \\ \text { O } & 1.775409 & 0.529241 & 3.319299 \\ \text { C } & 0.284701 & 3.016072 & 0.843572 \\ \text { O } & 0.929836 & 3.742424 & 0.232487 \\ \text { C } & -2.526629 & 2.292882 & 0.866479 \\ \text { O } & -3.439086 & 2.602844 & 0.238972 \\ \text { C } & -1.989803 & 0.236293 & 2.856943 \\ \text { O } & -2.614452 & -0.576000 & 3.372994\end{array}$




$\begin{array}{rrrrrrrr}\mathrm{O} & -0.848122 & 1.047899 & -1.261151 & \mathrm{H} & -2.005015 & 1.401266 & -4.408246 \\ \mathrm{~N} & -1.491999 & 0.453379 & -2.608903 & \mathrm{H} & -2.093743 & 2.418931 & -2.941514 \\ \mathrm{C} & -0.630819 & -0.616816 & -3.128275 & \mathrm{H} & -0.507882 & 1.987407 & -3.624618 \\ \mathrm{H} & -1.106757 & -1.065858 & -3.999399 & \mathrm{C} & -2.829890 & -0.033041 & -2.231617 \\ \mathrm{H} & 0.333753 & -0.194207 & -3.392929 & \mathrm{H} & -3.311972 & -0.467302 & -3.106725 \\ \mathrm{H} & -0.522166 & -1.363781 & -2.341329 & \mathrm{H} & -2.699408 & -0.788845 & -1.457404 \\ \mathrm{C} & -1.527533 & 1.651197 & -3.460995 & \mathrm{H} & -3.406079 & 0.803366 & -1.847243\end{array}$

\begin{tabular}{lrrrrrrr}
\multicolumn{7}{c}{$\mathrm{NMe}_{3}:$} & \multicolumn{2}{c}{$\mathrm{E}=-174.164957281 \mathrm{au}$} \\
\multicolumn{7}{c}{$\mathrm{ZPE}=0.11965096 \mathrm{au}$} \\
$\mathrm{N}$ & 0.135307 & 0.900264 & -1.918930 & $\mathrm{H}$ & 1.681641 & 2.281273 & -1.631761 \\
$\mathrm{C}$ & 0.928260 & 0.020893 & -2.761227 & $\mathrm{H}$ & 0.583868 & 2.710124 & -2.962408 \\
$\mathrm{H}$ & 0.892961 & 0.309231 & -3.827535 & $\mathrm{C}$ & -1.272566 & 0.846959 & -2.274555 \\
$\mathrm{H}$ & 1.971057 & 0.040794 & -2.440177 & $\mathrm{H}$ & -1.637991 & -0.177878 & -2.190326 \\
$\mathrm{H}$ & 0.562370 & -1.003688 & -2.675951 & $\mathrm{H}$ & -1.851024 & 1.474640 & -1.594614 \\
$\mathrm{C}$ & 0.638934 & 2.262881 & -1.953258 & $\mathrm{H}$ & -1.462912 & 1.194367 & -3.306334 \\
$\mathrm{H}$ & 0.059192 & 2.889866 & -1.273762 & & & &
\end{tabular}

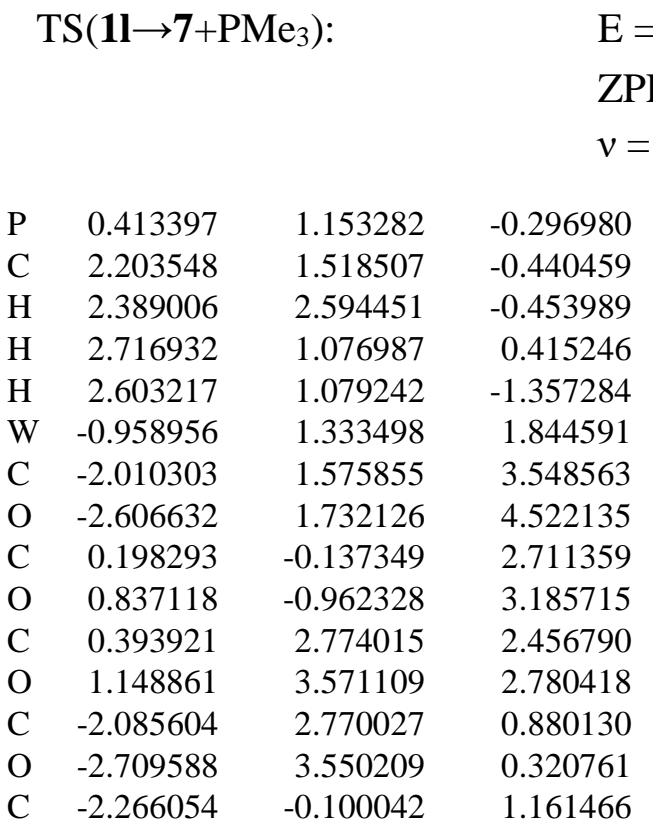

$\begin{array}{lrrr}\mathrm{O} & -2.991075 & -0.893535 & 0.755974 \\ \mathrm{O} & -0.239387 & 1.774367 & -1.602227 \\ \mathrm{P} & -1.050633 & 0.501305 & -2.967239 \\ \mathrm{C} & -0.679128 & -1.076897 & -2.225157 \\ \mathrm{H} & -1.540896 & -1.558128 & -1.766882 \\ \mathrm{H} & -0.202086 & -1.740807 & -2.942109 \\ \mathrm{H} & 0.030025 & -0.852090 & -1.382412 \\ \mathrm{C} & -0.900986 & 0.335420 & -4.786873 \\ \mathrm{H} & -1.660717 & -0.342455 & -5.186240 \\ \mathrm{H} & -1.023998 & 1.318118 & -5.241971 \\ \mathrm{H} & 0.090832 & -0.043240 & -5.031449 \\ \mathrm{C} & -2.759460 & 0.984153 & -2.635936 \\ \mathrm{H} & -3.462329 & 0.379888 & -3.211077 \\ \mathrm{H} & -2.959718 & 0.865354 & -1.572811 \\ \mathrm{H} & -2.887786 & 2.036393 & -2.892527\end{array}$

$\mathrm{PMe}_{3}$ :

$\begin{array}{rrrr}\mathrm{P} & 0.337680 & -0.364891 & 1.450076 \\ \mathrm{C} & 1.513517 & 0.864051 & 0.725328 \\ \mathrm{H} & 1.706307 & 1.655490 & 1.451595 \\ \mathrm{H} & 2.462616 & 0.373043 & 0.503751 \\ \mathrm{H} & 1.123121 & 1.312114 & -0.192461 \\ \mathrm{C} & 0.005488 & -1.394559 & -0.049135 \\ \mathrm{H} & -0.800329 & -2.098972 & 0.164066\end{array}$

$E=-460.428158985 \mathrm{au}$

$$
\mathrm{ZPE}=0.111962960 \mathrm{au}
$$

$=-174.164957281 \mathrm{au}$




\begin{tabular}{|c|c|c|c|c|c|c|}
\hline \multicolumn{2}{|l|}{$\mathrm{TS}(\mathbf{1 1} \rightarrow \mathbf{1 1})$} & $\begin{array}{l}\mathrm{E}= \\
\mathrm{ZP} \\
v=\end{array}$ & & & & \\
\hline-0.244982 & 0.967522 & -0.585193 & $\mathrm{O}$ & -2.818625 & -0.928358 & 1.905291 \\
\hline 1.523586 & 0.721022 & -0.982232 & $\mathrm{O}$ & -0.807489 & 1.981596 & -1.820240 \\
\hline 2.070829 & 1.638029 & -0.753133 & $\mathrm{P}$ & -1.460832 & 0.437711 & -2.312886 \\
\hline 1.917369 & -0.080556 & -0.355131 & $\mathrm{C}$ & -1.202498 & -1.386413 & -2.560659 \\
\hline 1.674852 & 0.467542 & -2.031504 & $\mathrm{H}$ & -1.848031 & -1.782910 & -3.348331 \\
\hline-0.799599 & 1.560281 & 1.783308 & $\mathrm{H}$ & -0.162642 & -1.569647 & -2.837689 \\
\hline-1.251154 & 2.121646 & 3.690017 & $\mathrm{H}$ & -1.402724 & -1.894751 & -1.617148 \\
\hline-1.500569 & 2.452458 & 4.760866 & C & -1.399532 & 0.926291 & -4.077810 \\
\hline 0.731124 & 0.345355 & 2.436850 & $\mathrm{H}$ & -1.990185 & 0.237900 & -4.682678 \\
\hline 1.586902 & -0.336846 & 2.777197 & $\mathrm{H}$ & -1.765560 & 1.944759 & -4.193793 \\
\hline 0.538704 & 3.122516 & 1.588999 & $\mathrm{H}$ & -0.363801 & 0.891665 & -4.419787 \\
\hline 1.294891 & 3.972270 & 1.460911 & $\mathrm{C}$ & -3.190874 & 0.521927 & -1.787886 \\
\hline-2.318094 & 2.736765 & 1.026883 & $\mathrm{H}$ & -3.809874 & 0.011092 & -2.526563 \\
\hline-3.159784 & 3.370991 & 0.580394 & $\mathrm{H}$ & -3.319638 & 0.032837 & -0.823914 \\
\hline-2.099265 & -0.035688 & 1.881673 & $\mathrm{H}$ & -3.499029 & 1.562396 & -1.708639 \\
\hline
\end{tabular}

11:

$\begin{array}{lrrr}\mathrm{P} & -0.472973 & 1.845969 & -0.931613 \\ \mathrm{C} & 1.263420 & 1.489896 & -1.418804 \\ \mathrm{H} & 1.896869 & 2.215954 & -0.906083 \\ \mathrm{H} & 1.570900 & 0.489899 & -1.108170 \\ \mathrm{H} & 1.398722 & 1.611547 & -2.493790 \\ \mathrm{~W} & -0.902888 & 1.535175 & 1.553789 \\ \mathrm{C} & -1.125299 & 1.467054 & 3.574947 \\ \mathrm{O} & -1.238611 & 1.437150 & 4.717715 \\ \mathrm{C} & 0.466921 & 0.011177 & 1.549509 \\ \mathrm{O} & 1.218113 & -0.856594 & 1.491449 \\ \mathrm{C} & 0.598193 & 2.943359 & 1.800722 \\ \mathrm{O} & 1.429469 & 3.714427 & 1.950442 \\ \mathrm{C} & -2.320442 & 3.034843 & 1.340664 \\ \mathrm{O} & -3.113291 & 3.843106 & 1.191327 \\ \mathrm{C} & -2.395426 & 0.146383 & 1.385663\end{array}$

$\mathrm{E}=-1548.962962590 \mathrm{au}$

$\mathrm{ZPE}=0.19796449 \mathrm{au}$

\section{$\mathrm{TS}(\mathbf{1 l} \rightarrow \mathbf{1 2}):$}

$$
\begin{aligned}
& E=-1548.905623739 \mathrm{au} \\
& \mathrm{ZPE}=0.19193272 \mathrm{au} \\
& v=-1096.15 \mathrm{~cm}^{-1}
\end{aligned}
$$

$\begin{array}{lrrr}\mathrm{P} & -0.062593 & 0.241432 & -0.246225 \\ \mathrm{C} & 1.741123 & 0.371879 & -0.550655 \\ \mathrm{H} & 2.080076 & 1.406604 & -0.488607 \\ \mathrm{H} & 2.255886 & -0.209621 & 0.216528 \\ \mathrm{H} & 1.999464 & -0.036543 & -1.529988 \\ \mathrm{~W} & -0.888412 & 1.264188 & 1.932671 \\ \mathrm{C} & -1.550925 & 2.138797 & 3.638094 \\ \mathrm{O} & -1.922696 & 2.652414 & 4.598903 \\ \mathrm{C} & 0.908538 & 0.774484 & 2.818134\end{array}$

$\begin{array}{lrrr}\mathrm{O} & 1.914895 & 0.493142 & 3.290452 \\ \mathrm{C} & -0.074275 & 3.025850 & 1.239046 \\ \mathrm{O} & 0.390193 & 3.984686 & 0.815480 \\ \mathrm{C} & -2.640367 & 1.692115 & 0.945509 \\ \mathrm{O} & -3.603374 & 1.914950 & 0.360695 \\ \mathrm{C} & -1.664373 & -0.562683 & 2.493834 \\ \mathrm{O} & -2.096354 & -1.582818 & 2.789401 \\ \mathrm{O} & -0.619148 & 1.257546 & -1.514267 \\ \mathrm{P} & -1.223512 & 0.604724 & -2.864624\end{array}$




$\begin{array}{lrrr}\mathrm{C} & -1.167841 & -1.096618 & -2.685517 \\ \mathrm{H} & -2.118433 & -1.600962 & -2.555107 \\ \mathrm{H} & -0.457623 & -1.625959 & -3.310081 \\ \mathrm{H} & -0.580451 & -0.965353 & -1.284851 \\ \mathrm{C} & -0.189275 & 1.257123 & -4.179856 \\ \mathrm{H} & -0.629935 & 1.034118 & -5.151279\end{array}$

$\begin{array}{rrrr}\mathrm{H} & -0.086286 & 2.336057 & -4.059360 \\ \mathrm{H} & 0.796554 & 0.795783 & -4.116498 \\ \mathrm{C} & -2.869143 & 1.306187 & -2.990763 \\ \mathrm{H} & -3.308639 & 1.060503 & -3.957090 \\ \mathrm{H} & -3.489098 & 0.896794 & -2.193333 \\ \mathrm{H} & -2.814165 & 2.388615 & -2.871120\end{array}$

12:

$$
E=-1548.935035772 \mathrm{au}
$$$$
\mathrm{ZPE}=0.19502338 \mathrm{au}
$$

$\begin{array}{lrrr}\mathrm{P} & -0.202017 & 0.274496 & -0.213984 \\ \mathrm{C} & 1.543902 & -0.160263 & -0.469992 \\ \mathrm{H} & 2.144661 & 0.749034 & -0.439347 \\ \mathrm{H} & 1.864073 & -0.818623 & 0.339445 \\ \mathrm{H} & 1.674740 & -0.662056 & -1.427852 \\ \mathrm{~W} & -0.857357 & 1.483246 & 1.877399 \\ \mathrm{C} & -1.407201 & 2.523629 & 3.542872 \\ \mathrm{O} & -1.718854 & 3.116206 & 4.474938 \\ \mathrm{C} & 0.798847 & 0.720623 & 2.842413 \\ \mathrm{O} & 1.722338 & 0.285198 & 3.361715 \\ \mathrm{C} & 0.271922 & 3.071370 & 1.197344 \\ \mathrm{O} & 0.906102 & 3.939879 & 0.803767 \\ \mathrm{C} & -2.488520 & 2.193544 & 0.833899 \\ \mathrm{O} & -3.384526 & 2.571833 & 0.228758 \\ \mathrm{C} & -1.958940 & -0.154871 & 2.478851\end{array}$

$\begin{array}{rrrr}\mathrm{O} & -2.566284 & -1.073448 & 2.792534 \\ \mathrm{O} & -0.601896 & 1.121852 & -1.545885 \\ \mathrm{P} & -1.111136 & 0.485608 & -3.028634 \\ \mathrm{C} & -0.617387 & -1.007868 & -3.516974 \\ \mathrm{H} & -1.177247 & -1.882610 & -3.219231 \\ \mathrm{H} & 0.375533 & -1.148731 & -3.920406 \\ \mathrm{H} & -0.808972 & -0.976102 & -0.466592 \\ \mathrm{C} & -0.520450 & 1.840959 & -4.056567 \\ \mathrm{H} & -1.011026 & 1.802781 & -5.027902 \\ \mathrm{H} & -0.716682 & 2.797285 & -3.573641 \\ \mathrm{H} & 0.555162 & 1.727618 & -4.196785 \\ \mathrm{C} & -2.897274 & 0.590835 & -2.808399 \\ \mathrm{H} & -3.396522 & 0.508949 & -3.772418 \\ \mathrm{H} & -3.222222 & -0.233831 & -2.172365 \\ \mathrm{H} & -3.162955 & 1.530895 & -2.327438\end{array}$

$\mathrm{TS}\left(\mathbf{1 m} \rightarrow \mathbf{7}+\mathrm{SMe}_{2}\right):$

$\begin{array}{lr}\mathrm{P} & -0.040686 \\ \mathrm{C} & 1.787907 \\ \mathrm{H} & 2.323366 \\ \mathrm{H} & 2.019499 \\ \mathrm{H} & 2.131782 \\ \mathrm{~W} & -0.908156 \\ \mathrm{C} & -1.495286 \\ \mathrm{O} & -1.818998 \\ \mathrm{C} & 0.490647 \\ \mathrm{O} & 1.271371 \\ \mathrm{C} & 0.499085 \\ \mathrm{O} & 1.287936 \\ \mathrm{C} & -2.283264\end{array}$

\begin{abstract}
0.116612
$-0.142396$

0.796817

$-0.813525$

$-0.611623$

1.534458

2.616878

3.245892

0.553553

$-0.002888$

2.949163

3.720837

2.436548
\end{abstract}

$$
\begin{aligned}
& E=-1565.817787309 \mathrm{au} \\
& \mathrm{ZPE}=0.15813856 \mathrm{au} \\
& v=-442.26 \mathrm{~cm}^{-1}
\end{aligned}
$$
$-0.154615$
$-0.124542$
0.020290
0.705127
$-1.048739$
1.850679
3.443351
4.353717
3.005012
3.633841
1.324678
1.016336
0.614379

$\mathrm{SMe}_{2}$ :

$\begin{array}{lr}\mathrm{S} & -1.089431 \\ \mathrm{C} & -0.802733 \\ \mathrm{H} & -1.662411 \\ \mathrm{H} & 0.064606\end{array}$

$\begin{array}{ll}-0.262516 & -3.280297 \\ -1.477433 & -1.965807 \\ -2.138931 & -1.853948 \\ -2.068130 & -2.257852\end{array}$

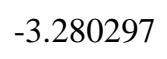

$\begin{array}{lrrr}\mathrm{O} & -3.044615 & 2.909623 & -0.103700 \\ \mathrm{C} & -2.279598 & 0.055002 & 2.266046 \\ \mathrm{O} & -3.043282 & -0.773378 & 2.481149 \\ \mathrm{O} & -0.157808 & 1.212678 & -1.397157 \\ \mathrm{~S} & -0.747227 & 0.656404 & -3.063717 \\ \mathrm{C} & -0.336775 & -1.061711 & -3.054218 \\ \mathrm{H} & -1.126868 & -1.643515 & -3.522708 \\ \mathrm{H} & 0.615923 & -1.203056 & -3.557225 \\ \mathrm{H} & -0.276436 & -1.337266 & -1.973045 \\ \mathrm{C} & -2.479976 & 0.566005 & -2.619861 \\ \mathrm{H} & -3.037790 & 0.134471 & -3.449414 \\ \mathrm{H} & -2.568199 & -0.054309 & -1.725885 \\ \mathrm{H} & -2.815496 & 1.580505 & -2.418044\end{array}$

$$
\begin{aligned}
& E=-477.404491839 \mathrm{au} \\
& Z P E=0.07529542 \mathrm{au}
\end{aligned}
$$

$-1.640074$ 


$\begin{array}{lrrr} & \mathrm{TS}(\mathbf{1 m} \rightarrow \mathbf{1 3}): & & \mathrm{E}= \\ & & & \mathrm{ZPE} \\ & & & v=-4 \\ & & & \\ \mathrm{P} & -0.409349 & 0.428710 & -0.035577 \\ \mathrm{C} & 0.919478 & -0.815094 & 0.120199 \\ \mathrm{H} & 1.859796 & -0.304532 & 0.341501 \\ \mathrm{H} & 0.683335 & -1.479937 & 0.952440 \\ \mathrm{H} & 1.042930 & -1.401296 & -0.790085 \\ \mathrm{~W} & -0.800518 & 1.950459 & 1.896880 \\ \mathrm{C} & -1.049692 & 3.294429 & 3.412496 \\ \mathrm{O} & -1.171061 & 4.061501 & 4.257745 \\ \mathrm{C} & 0.111923 & 0.593462 & 3.148040 \\ \mathrm{O} & 0.622850 & -0.181585 & 3.820554 \\ \mathrm{C} & 1.042183 & 2.774887 & 1.455821 \\ \mathrm{O} & 2.069044 & 3.215319 & 1.207904 \\ \mathrm{C} & -1.709040 & 3.224097 & 0.546450\end{array}$

$$
\begin{aligned}
& E=-1565.809315449 \mathrm{au} \\
& Z P E=0.15809850 \mathrm{au} \\
& v=-444.35 \mathrm{~cm}^{-1}
\end{aligned}
$$

$\begin{array}{lrrr}\mathrm{O} & -2.209849 & 3.911911 & -0.218767 \\ \mathrm{C} & -2.629460 & 1.076278 & 2.306505 \\ \mathrm{O} & -3.638281 & 0.582323 & 2.528257 \\ \mathrm{O} & 0.002754 & 1.207791 & -1.514992 \\ \mathrm{~S} & -1.392539 & 0.300197 & -2.035592 \\ \mathrm{C} & -0.797114 & 0.488415 & -3.779157 \\ \mathrm{H} & -1.587016 & 0.125955 & -4.436621 \\ \mathrm{H} & -0.619400 & 1.547676 & -3.944426 \\ \mathrm{H} & 0.121313 & -0.076583 & -3.932862 \\ \mathrm{C} & -1.591995 & -1.514767 & -2.181306 \\ \mathrm{H} & -2.153614 & -1.720427 & -3.093351 \\ \mathrm{H} & -0.621335 & -2.005880 & -2.236988 \\ \mathrm{H} & -2.128286 & -1.841531 & -1.293331\end{array}$

13:

$$
\begin{aligned}
& E=-1565.919947009 \mathrm{au} \\
& Z P E=0.16061919 \mathrm{au}
\end{aligned}
$$

$\begin{array}{lrrr}\mathrm{P} & 0.163347 & 1.518672 & -0.873866 \\ \mathrm{C} & 1.923864 & 1.028084 & -0.937481 \\ \mathrm{H} & 2.489284 & 1.783602 & -0.387373 \\ \mathrm{H} & 2.079947 & 0.062676 & -0.453368 \\ \mathrm{H} & 2.277570 & 0.999949 & -1.967644 \\ \mathrm{~W} & -0.888906 & 1.467983 & 1.408186 \\ \mathrm{C} & -1.566490 & 1.633901 & 3.323614 \\ \mathrm{O} & -1.933496 & 1.734184 & 4.405448 \\ \mathrm{C} & 0.157569 & -0.243876 & 1.833091 \\ \mathrm{O} & 0.751356 & -1.206779 & 2.028130 \\ \mathrm{C} & 0.736511 & 2.618500 & 1.968605 \\ \mathrm{O} & 1.628565 & 3.252307 & 2.300266 \\ \mathrm{C} & -1.914944 & 3.170160 & 0.803915\end{array}$

$\begin{array}{lrrr}\mathrm{O} & -2.483090 & 4.093631 & 0.449848 \\ \mathrm{C} & -2.573412 & 0.367355 & 0.978364 \\ \mathrm{O} & -3.531436 & -0.234086 & 0.783056 \\ \mathrm{O} & -0.117445 & 2.592408 & -1.876287 \\ \mathrm{~S} & -0.476357 & -0.367238 & -2.275420 \\ \mathrm{C} & -0.772987 & -1.791022 & -1.208633 \\ \mathrm{H} & -1.320998 & -2.544384 & -1.771324 \\ \mathrm{H} & 0.199336 & -2.184989 & -0.920761 \\ \mathrm{H} & -1.330268 & -1.493721 & -0.325189 \\ \mathrm{C} & -2.148654 & 0.203978 & -2.636680 \\ \mathrm{H} & -2.629247 & -0.506695 & -3.305290 \\ \mathrm{H} & -2.717724 & 0.317051 & -1.717861 \\ \mathrm{H} & -2.034838 & 1.170126 & -3.123612\end{array}$

\section{$\mathrm{TS}(\mathbf{1 m} \rightarrow \mathbf{1 4}):$}
0.428710
$-0.035577$
$-0.815094$
0.120199
$-0.304532$
$-1.479937$
$-1.401296$
1.950459
3.294429
4.061501
0.593462
$-0.181585$$$
0.952440
$$$$
-0.790085
$$$$
1.896880
$$$$
3.412496
$$$$
4.257745
$$$$
3.148040
$$$$
3.820554
$$

$$
\begin{aligned}
& \mathrm{E}= \\
& \mathrm{ZP} \\
& v= \\
& \\
& 577 \\
& 199 \\
& 501
\end{aligned}
$$

C

2.774887

1.455821

O 2.069044

3.215319

3.224097

1.207904

C $\quad-1.709040$

3.911911

1.076278

0.582323

1.207791

0.300197

0.488415

0.125955
0.546450

$-0.218767$

2.306505

2.528257

$-1.514992$

$-2.035592$

$-3.779157$

$-4.436621$ 


$\begin{array}{lrrrrrrr}\mathrm{H} & -0.619400 & 1.547676 & -3.944426 & \mathrm{H} & -2.153614 & -1.720427 & -3.093351 \\ \mathrm{H} & 0.121313 & -0.076583 & -3.932862 & \mathrm{H} & -0.621335 & -2.005880 & -2.236988 \\ \mathrm{C} & -1.591995 & -1.514767 & -2.181306 & \mathrm{H} & -2.128286 & -1.841531 & -1.293331\end{array}$

14:

$\begin{array}{lrrr}\text { P } & 0.084687 & -0.212547 & -0.443635 \\ \mathrm{C} & 0.534293 & -0.525696 & 1.339259 \\ \mathrm{H} & 1.624814 & -0.565191 & 1.355373 \\ \mathrm{H} & 0.219584 & 0.269186 & 2.013925 \\ \mathrm{H} & 0.161765 & -1.487744 & 1.690962 \\ \mathrm{~W} & 0.981975 & 2.129840 & -1.129888 \\ \mathrm{C} & 1.915737 & 3.835638 & -1.669566 \\ \mathrm{O} & 2.460690 & 4.803983 & -1.970242 \\ \mathrm{C} & -0.810860 & 3.041382 & -1.569939 \\ \mathrm{O} & -1.787396 & 3.586096 & -1.827529 \\ \mathrm{C} & 0.749391 & 2.766842 & 0.819556 \\ \mathrm{O} & 0.600052 & 3.108850 & 1.901855 \\ \mathrm{C} & 2.829247 & 1.329256 & -0.676410\end{array}$

$\mathrm{E}=-1565.839033329 \mathrm{au}$

$\mathrm{ZPE}=0.16133737 \mathrm{au}$

$\begin{array}{lrrr}\mathrm{O} & 3.862532 & 0.905781 & -0.418245 \\ \mathrm{C} & 1.078845 & 1.392642 & -3.049972 \\ \mathrm{O} & 1.105436 & 0.971058 & -4.116120 \\ \mathrm{O} & -2.449510 & 1.291602 & 0.753294 \\ \mathrm{~S} & -2.007669 & 0.143577 & -0.041523 \\ \mathrm{C} & -2.758344 & 0.168279 & -1.663618 \\ \mathrm{H} & -3.814587 & 0.388301 & -1.517895 \\ \mathrm{H} & -2.279729 & 0.959030 & -2.233666 \\ \mathrm{H} & -2.608465 & -0.796530 & -2.141707 \\ \mathrm{C} & -2.712496 & -1.341796 & 0.676853 \\ \mathrm{H} & -3.793359 & -1.212249 & 0.697399 \\ \mathrm{H} & -2.418483 & -2.204388 & 0.084281 \\ \mathrm{H} & -2.323309 & -1.413284 & 1.689848\end{array}$

$$
\mathrm{TS}\left(\mathbf{1 0} \rightarrow 7+\mathrm{N}_{2}\right):
$$

$\begin{array}{lrrr} & \mathrm{TS}\left(\mathbf{1 0} \rightarrow \mathbf{7}+\mathrm{N}_{2}\right): & \mathrm{E}= \\ & & & \mathrm{ZPE} \\ & & & \\ & & & \\ & & & \\ \mathrm{P} & -0.006034 & 0.006784 & -0.005510 \\ \mathrm{C} & 1.824328 & 0.009384 & 0.015669 \\ \mathrm{H} & 2.229959 & 1.020839 & 0.010476 \\ \mathrm{H} & 2.145392 & -0.498037 & 0.928781 \\ \mathrm{H} & 2.236778 & -0.554146 & -0.832200 \\ \mathrm{~W} & -0.938423 & -2.288773 & -0.176841 \\ \mathrm{C} & -1.729736 & -4.182250 & -0.302376 \\ \mathrm{O} & -2.157707 & -5.240954 & -0.385350 \\ \mathrm{C} & 0.645199 & -2.921295 & -1.343410 \\ \mathrm{O} & 1.527190 & -3.306232 & -1.962692\end{array}$

$E=-1197.671752142 \mathrm{au}$

$\mathrm{ZPE}=0.08831495 \mathrm{au}$ $v=-684.12 \mathrm{~cm}^{-1}$

\footnotetext{
$\mathrm{N}_{2}$ :

$E=-109.376287850 \mathrm{au}$

$\mathrm{ZPE}=0.00558589 \mathrm{au}$

$\begin{array}{llll}\mathrm{N} & 1.349971 & -1.710311 & -0.260960\end{array}$

$\begin{array}{llll}\mathrm{N} & 0.793127 & -2.622855 & -0.043996\end{array}$ $-0.260960$
}

$\begin{array}{lrrr}\mathrm{C} & 0.188590 & -2.794091 & 1.479880 \\ \mathrm{O} & 0.837817 & -3.052251 & 2.385782 \\ \mathrm{C} & -2.526239 & -1.702881 & 1.023823 \\ \mathrm{O} & -3.406538 & -1.390913 & 1.681415 \\ \mathrm{C} & -2.012628 & -1.692760 & -1.840505 \\ \mathrm{O} & -2.587943 & -1.335259 & -2.761845 \\ \mathrm{O} & -0.174656 & 0.963481 & -1.637773 \\ \mathrm{~N} & 0.484111 & 0.478828 & -2.671156 \\ \mathrm{~N} & 1.289258 & -0.156570 & -3.128445\end{array}$

